\title{
Anomaly-free cosmological perturbations \\ in effective canonical quantum gravity
}

\author{
Aurelien Barrau, ${ }^{a}$ Martin Bojowald, ${ }^{b}$ Gianluca Calcagni, ${ }^{c}$ Julien \\ Grain, ${ }^{d}$ Mikhail Kagan ${ }^{b, e}$
}

${ }^{a}$ Laboratoire de Physique Subatomique et de Cosmologie, Université Grenoble-Alpes, CNRS/IN2P3 53 av. des Martyrs, 38025 Grenoble cedex, France

${ }^{b}$ Institute for Gravitation and the Cosmos, The Pennsylvania State University, 104 Davey Lab, University Park, PA 16802, USA

${ }^{c}$ Instituto de Estructura de la Materia, CSIC, Serrano 121, 28006 Madrid, Spain

${ }^{d}$ Institut d'Astrophysique Spatiale, CNRS/INSU, Université Paris-Sud 11, Orsay F-91405 France

${ }^{e}$ Division of Science and Engineering, The Pennsylvania State University, Abington, 1600 Woodland Road, Abington, PA 19116, USA

E-mail: barrau@in2p3.fr, bojowald@gravity.psu.edu, calcagni@iem.cfmac.csic.es, julien.grain@ias.u-psud.fr, mak411@psu.edu

Abstract. This article lays out a complete framework for an effective theory of cosmological perturbations with corrections from canonical quantum gravity. Since several examples exist for quantum-gravity effects that change the structure of space-time, the classical perturbative treatment must be rethought carefully. The present discussion provides a unified picture of several previous works, together with new treatments of higher-order perturbations and the specification of initial states.

Keywords: Quantum cosmology, Cosmology of theories beyond the SM, cosmological perturbation theory 


\section{Contents}

1 Introduction $\quad 2$

2 Canonical quantum gravity $\quad 6$

2.1 Classical theory 6

2.2 Quantization 8

2.3 Quantum corrections 10

$\begin{array}{lll}\text { 2.3.1 Degrees of freedom and effective phase space } & 10\end{array}$

$\begin{array}{ll}\text { 2.3.2 Inverse-triad corrections } & 13\end{array}$

$\begin{array}{lll}\text { 2.3.3 Holonomy corrections and their ambiguities } & 14\end{array}$

2.3.4 Relation to homogeneous quantum cosmology (mini-superspace models) 14

3 Constraint algebra and space-time structure $\quad \mathbf{1 5}$

$\begin{array}{lll}3.1 & \text { Isolated modifications } & 15\end{array}$

$\begin{array}{ll}3.2 & \text { Examples of anomaly-free constraints } \\ & 3.2 .16\end{array}$

$\begin{array}{lll}3.2 .1 & \text { Spherical symmetry } & 17\end{array}$

$\begin{array}{ll}3.2 .2 \text { Cosmological perturbations } & 18\end{array}$

$\begin{array}{ll}3.2 .3 \text { Comparison } & 18\end{array}$

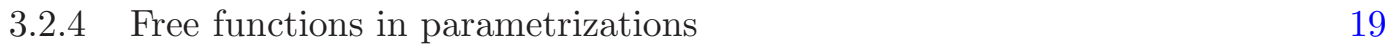

$\begin{array}{lll}3.3 & \text { Perturbation scheme } & 20\end{array}$

3.4 Deformed general relativity 23

$\begin{array}{lll}3.4 .1 & \text { Deformations } & 23\end{array}$

$\begin{array}{ll}3.4 .2 & \text { Evaluation of equations }\end{array}$

$\begin{array}{ll}3.4 .3 & \text { Space-time }\end{array}$

3.4.4 Signature change 28

3.4.5 Phase space and the Hojman-Kuchař-Teitelboim theorem 30

$\begin{array}{ll}3.4 .6 & \text { Possible observations }\end{array}$

3.5 Add-on inhomogeneity 32

3.5.1 Hybrid models 32

3.5.2 Dressed metric 33

$\begin{array}{lll}\text { 3.5.3 Relation to possible observations } & 34\end{array}$

4 States $\quad 35$

$\begin{array}{lll}4.1 & \text { Inflaton vacuum } & 35\end{array}$

4.2 Quantum-gravity state 36

$\begin{array}{lll}5 & \text { Conclusions } & 37\end{array}$

$\begin{array}{ll}\text { A Canonical effective theory } & 38\end{array}$

B Consistency of inverse-triad corrected equations 39 


\section{Introduction}

One of the most promising avenues towards tests of quantum gravity is the study of the corrections it implies in cosmological perturbation equations. With some luck, characteristic traces of high-energy space-time phenomena may be left in the observable part of the cosmic microwave background. The derivation of power spectra from different approaches to quantum gravity is therefore one of the most active areas in the field. In this article, we consider the status of developments in canonical quantum gravity, in particular loop quantum gravity.

Canonical quantum gravity does not straightforwardly lead to cosmological perturbation equations. Given a consistent quantum theory, one would have to find a suitable class of semiclassical states, so that the expectation values they define for basic operators (such as the spatial metric or its inhomogeneous modes) are subject to equations of motion derived from the Wheeler-DeWitt equation. The issue of finding semi-classical (or other suitable) states in canonical quantum gravity is tricky - for one, we are unaware of any ground state that would be a good starting point for a perturbative expansion. And if we assume that the problem can be solved, consistent cosmological perturbation equations do not follow automatically, for reasons closely related to the generally covariant nature and complicated gauge content of the theory.

The classical theory has more equations than unknowns, and they appear as different types. There are constraints that depend only on the modes and their first time derivatives (or on configuration variables and their canonically conjugate momenta), and there are equations of motion that determine second-order time derivatives. The classical set of equations satisfies two important consistency conditions: (i) The constraints, a spatial function $C$ and a vector field $\vec{D}$, are preserved by the equations of motion, so that they need to be imposed only for initial values and then automatically hold at all later times. This preservation of the constraints is the reason why the set of equations for a smaller number of free functions is consistent and allows the right number of non-trivial solutions. (ii) The constraints are generators of a large set of gauge transformations which include space-time Lie derivatives of phase-space functions, in particular their time derivatives. Time derivatives of momentum variables imply the second-order equations of motion; by being generated by gauge-inducing constraints, they automatically preserve the constraints.

In canonical language, the equations of motion are generated by the constraints, that is they follow from equations $\dot{f}=\{f, H[N, \vec{M}]\}$ in which the time derivative (along a direction with space-time components related to the lapse function $N$ and the shift vector $\vec{M}$ ) of a mode function $f$ is given by the Poisson bracket with a Hamiltonian $H[N, \vec{M}]$. This Hamiltonian is a linear combination $H[N, \vec{M}]=\int(N C+\vec{M} \cdot \vec{D})$ of the constraint functions $C$ and $\vec{D}$ and therefore must vanish when evaluated for solutions of the field equations, for all $N$ and $\vec{M}$. Property (i) is guaranteed if $0=\dot{H}\left[N_{1}, \vec{M}_{1}\right]=\left\{H\left[N_{1}, \vec{M}_{1}\right], H[N, \vec{M}]\right\}$ for all $N_{1}$ and $\vec{M}_{1}$ and for all configurations for which $H\left[N_{1}, \vec{M}_{1}\right]=0$, in which case the constraints are called first-class. The gauge transformations mentioned in property (ii) take the form $\delta_{\left(\epsilon_{0}, \vec{\epsilon}\right.} f=\left\{f, H\left[\epsilon_{0}, \vec{\epsilon}\right]\right\}$. For the specific choice of $\left(\epsilon_{0}, \vec{\epsilon}\right)=(N, \vec{M})$, one obtains time derivatives as gauge transformations. The general gauge transformation with parameters $\left(\epsilon_{0}, \vec{\epsilon}\right)$ amounts to a Lie derivative along a space-time vector field with components $\left(\epsilon_{0} / N, \vec{\epsilon}-\epsilon_{0} \vec{M} / N\right)$. Property (ii) then has the following consequence: By choosing different linear combinations, varying $N$ and $\vec{M}$ in the Hamiltonian, one takes into account all possible time choices. In other words, the theory is invariant under reparametrizations of time. It is therefore possible to express the equations of motion in terms of only gauge-invariant (i.e., coordinate 
independent) combinations of the modes. No gauge artifacts couple to the physical degrees of freedom.

These consistency conditions are a specific realization of the general concept of a firstclass system of constraints, using the notation introduced by Dirac. There is a set of constraints, in our case $H[N, \vec{M}]$, or individually $C[N]:=\int N C=0$ and $\vec{D}[\vec{M}]:=\int \vec{M} \cdot \vec{D}=0$, so that their Poisson brackets $\left\{\vec{D}\left[\vec{M}_{1}\right], \vec{D}\left[\vec{M}_{2}\right]\right\},\{C[N], \vec{D}[\vec{M}]\}$ and $\left\{C\left[N_{1}\right], C\left[N_{2}\right]\right\}$ vanish whenever the constraints are satisfied. In the case of gravity, the constraints obey the hypersurface-deformation algebra [1]

$$
\begin{aligned}
\left\{\vec{D}\left[\vec{M}_{1}\right], \vec{D}\left[\vec{M}_{2}\right]\right\} & =-\vec{D}\left[\mathcal{L}_{\vec{M}_{2}} \vec{M}_{1}\right], \\
\{C[N], \vec{D}[\vec{M}]\} & =-C\left[\mathcal{L}_{\vec{M}} N\right], \\
\left\{C\left[N_{1}\right], C\left[N_{2}\right]\right\} & =\vec{D}\left[N_{1} \vec{\nabla} N_{2}-N_{2} \vec{\nabla} N_{1}\right],
\end{aligned}
$$

and are first-class (here, a metric is used to obtain the contravariant derivative $\vec{\nabla}$ ). The preservation of the constraints $\dot{C}\left[N_{1}\right]=\left\{C\left[N_{1}\right], H[N, \vec{M}]\right\}$ and $\dot{\vec{D}}\left[\vec{M}_{1}\right]=\left\{\vec{D}\left[\vec{M}_{1}\right], H[N, \vec{M}]\right\}$ then follows directly, and the gauge-invariant modes $\phi$ are those that satisfy $\left\{\phi, H\left[\epsilon_{0}, \vec{\epsilon}\right]\right\}=0$ (for all $\epsilon_{0}$ and $\vec{\epsilon}$ ) whenever the constraints hold. The relations (1.1)-(1.3) have an interesting geometrical meaning [2]: They realize commutators of deformations of spacelike hypersurfaces in space-time along vector fields $N \vec{n}+\vec{M}$ with unit normals $\vec{n}$ to the hypersurfaces. Although this algebra does not refer to the dynamics of the theory, it has a tight relation with it: Second-order field equations for the metric which are covariant under symmetries obeying the algebra (1.1)-(1.3) must equal Einstein's equation (with an unrestricted cosmological constant) $[2,3]$. This result leaves only little room for quantum corrections. It is the canonical analog of the familiar statement that a local covariant action can correct the Einstein-Hilbert term only by higher-curvature contributions.

When quantum corrections are inserted in the equations of gravity, in particular in cosmological perturbation equations, it is never clear whether the delicate consistency conditions summarized in the first-class nature of the constraint algebra remain intact. Especially background-independent frameworks cannot directly rely on standard covariance arguments because their notion of space-time, encoded in (1.1)-(1.3), is supposed to emerge in some way from solutions to their equations. The consistency of the equations, however, must be ensured before they can be solved. If the approach used as well as the methods employed to derive semi-classical equations are covariant, the preserved symmetry implies consistent equations based on first-class constraints. This statement could, for instance, apply to effective equations derived from a path-integral quantization of gravity, provided one uses the correct integration measure to make the theory anomaly-free. (The latter condition is highly non-trivial.) In canonical quantum gravity, however, consistency must be shown explicitly because the different treatments of time and space derivatives in canonical quantizations eliminate manifest covariance.

In our discussion so far we have assumed that the full field content of gravity is considered without fixing the space-time gauge. The issues we mention can formally be circumvented if one fixes the gauge before quantizing or before inserting quantum corrections in effective equations. In many cases, gauge fixing before quantization can indeed be assumed to be harmless, but the situation considered here is different. First, the constraints we are dealing with are more complicated functions than, say, the Gauss constraint of Yang-Mills theories. It is therefore more likely that the constraints receive significant quantum corrections. If the constraints are quantum corrected, the gauge transformations they generate are not of 
the classical form. Gauge fixing before quantization is then inconsistent, because one would fix the gauge according to transformations which subsequently will be modified. Secondly, in the present case the dynamics is part of the gauge system. A consistent theory must therefore quantize gauge transformations and the dynamics at the same time; one cannot fix one part (the gauge) in order to derive the second part (the dynamics) in an unrestricted way. Therefore, in canonical quantum gravity one must consider the full space-time gauge algebra without restrictions, or else one cannot be sure that the resulting theory is consistent.

We should note that a less severe point of view, sometimes advocated in the literature, is to regard gauge fixing prior to quantization as part of the definition of a quantum theory which may turn out to be inequivalent to a non-gauge fixed quantum theory. In all cases where such a model can be checked to be self-consistent (as partially done in the hybrid cases mentioned in section 3.5.1), this attitude is legitimate. The resulting dynamics and physical predictions are, in general, quantitatively different from the theory quantized without fixing the gauge. Whenever available, however, the latter should be preferred because it implements the full system.

Another method that avoids dealing with the full system (1.1)-(1.3) is reduced phasespace quantization, often combined with a technique called deparametrization. (Examples for cosmological perturbations have been provided in [4-7].) For the reduced phase space, one solves the constraints classically and computes all observables invariant under gauge transformations. The resulting phase space is then to be quantized, which in general can be very complicated but may be possible in reduced or perturbative models. To facilitate the construction of gauge-invariant observables in a relational form, deparametrization selects a suitable phase-space degree of freedom to play the role of time. Formally, such a procedure leads to consistent equations, but they correspond to a quantized dynamics for classical observables rather than a complete quantization of the original gauge system. Moreover, for meaningful results one should check that the choice of internal time does not affect predictions, a form of covariance problem which is rarely analyzed in this context (see [8]).

The preferred method in our view is to quantize the constraints without classical specifications of gauge or observables. Canonical quantum gravity then provides operator versions of the classical constraints $C[N]$ and $\vec{D}[\vec{M}]$. Consistency is formulated just as for the classical constraints, except that commutators are used instead of Poisson brackets to define a first-class system. Making sure that such an operator algebra is first class is even more difficult than showing this property for a classical Poisson-bracket algebra because the result is usually sensitive to the particular factor ordering or possible regularization schemes used to define the operators. (A closed algebra may even be in conflict with Hermitian constraint operators $[9,10]$.) It is therefore useful to combine the derivation of a consistent algebra with the one of effective or semi-classical equations. Such an approach cannot prove that any particular theory of quantum gravity is consistent, due to the more-involved nature of operator algebras. But it can show what kind of geometrical or physical implications quantized constraints can have, including the potential for observational tests in cosmology.

With such an effective viewpoint, one (i) takes the corrections suggested by operator definitions in some approach to quantum gravity, (ii) parametrizes them so as to allow for sufficient freedom to encompass the ambiguities and unknowns in quantum operators, (iii) inserts them in the classical constraints and (iv) computes their algebra under Poisson brackets. In most cases, the algebra will no longer be first class, but careful choices of the functions and parameters used to specify the modifications may respect this important feature. If no choice of parameters gives rise to a first-class algebra, the quantum effect described by them 
is likely to be inconsistent. If there are consistent choices (which, in general, are not unique; see section 3.2.4), it is possible for the quantum effect studied to be part of an anomalyfree theory of quantum gravity, and one obtains a consistent model whose equations can be analyzed for further implications. In particular, one can then derive a complete set of cosmological perturbation equations and observables, incorporating quantum corrections. (At this stage, consistency being assured, one may use gauge fixing of the quantum-corrected constrained system to simplify solving the equations of motion.)

The prescription just sketched has been defined and evaluated in detail in [11], with applications to a certain type of corrections suggested by loop quantum gravity. In these cases, the algebra of constraints remained first class, but it was deformed: Its structure functions in (1.3), not just the constraints themselves, were affected by quantum corrections. Two developments that have happened in the meantime motivate us to take a renewed look: First, canonical effective theories and especially the origin of higher time derivatives in them has been better understood. Secondly, applied to a different type of modifications in loop quantum gravity [12,13], the surprising (and in some eyes, shocking) possibility of signature change [14] at high density was found. As a consequence, the framework has been subjected to enhanced scrutiny and criticism. This article presents a unified setting, combining insights found in several papers since [11], fleshing out some details that may so far have been mentioned only rather implicitly, and contrasting the approach with others that avoid dealing with the constraint algebra and instead use gauge fixing or deparametrization before the theory is quantized or modified.

We end this introduction with a summary of our main results. This article includes several passages of review in order to make especially the conceptual part of our discussions self-contained. A clear-cut separation between review material and novel statements would be rather artificial, since both are interwoven and the former is systematically utilized to introduce the latter. For the reader's convenience, however, we state here the main results in a nutshell:

1. The role of quantum moments in the effective dynamics is clarified and contrasted with that of holonomy modifications. The main difference is that higher-order time derivatives arise only by the first type of corrections (section 2.3). Section 2.3.1 contains a brief summary of effective methods, with special emphasis on the anomaly problem and the generality of this type of effective constructions. The techniques of relevance for our considerations do not make use of further approximations, such as derivative expansions, which would restrict the availability of effective descriptions.

2. By performing a detailed comparison of the most recent results on anomaly-free realizations of spherically symmetric models of loop quantum gravity as well as cosmological perturbations, we arrive at a simplified and more uniform picture of parametrizations of quantum corrections (section 3.2, especially sections 3.2.3 and 3.2.4).

3. The definition and possibility of signature change, which is an important ingredient for understanding the early universe, is discussed at length in section 3.4.4. In particular, we show that signature change, if it occurs, is not a consequence of perturbative inhomogeneity but rather of holonomy modifications used crucially in the background dynamics of loop quantum cosmology. We also contrast signature change with the more common phenomenon of instability. 
4. Details are provided for systematic extensions of perturbation schemes to higher orders, paying due attention to degrees of freedom and symplectic structures (section 3.3). The role of gauge invariance and different applications of gauge fixing or deparametrization are discussed across various approaches to cosmological perturbations (section 3.5).

5. When computing the inflationary spectra, the choice of vacuum is simply a choice of initial conditions consistent with the canonical effective equations. It is not a "requantization" of the theory, as sometimes hinted at in the literature. Our discussion of this question clarifies this issue and has the additional advantage of generalizing the specification of vacuum initial conditions to non-standard space-time structures as encountered in models of loop quantum gravity (section 4.1).

\section{Canonical quantum gravity}

Canonically, general relativity is described by the phase space $\left(h_{a b}, p^{a b}\right)$ of spatial metrics $h_{a b}$ and momenta $p^{a b}$ related to extrinsic curvature. The theory can then be parametrized conveniently using the ADM decomposition [15]. A space-time solution is

$$
\mathrm{d} s^{2}=-N^{2} \mathrm{~d} t^{2}+h_{a b}\left(\mathrm{~d} x^{a}+M^{a} \mathrm{~d} t\right)\left(\mathrm{d} x^{b}+M^{b} \mathrm{~d} t\right)
$$

where $h_{a b}$ changes between $t=$ const slices by $\mathcal{L}_{t} h_{a b}=\left\{h_{a b}, H[N, \vec{M}]\right\}$ together with $\mathcal{L}_{t} p^{a b}=$ $\left\{p^{a b}, H[N, \vec{M}]\right\}$. Initial values for $h_{a b}$ and $p^{a b}$ must obey the constraints $H[N, \vec{M}]=0$. Time derivatives are represented as Lie derivatives along the time-evolution vector field $t^{a}=N n^{a}+$ $M^{a}$ with the unit normal $n^{a}$. Since all equations are invariant under the transformations generated by $H\left[\epsilon_{0}, \vec{\epsilon}\right]$ and (1.1)-(1.3) imply that gauge transformations of fields obeying the constraints are space-time Lie derivatives, (2.1) is invariant under changes of coordinates.

Alternatively, one may use different variables that describe the same phase space after a canonical transformation (and perhaps an enlargement by further gauge degrees of freedom), such as Schwinger's [16] $\left(K_{a}^{i}, E_{j}^{b}\right)$ with the densitized triad $E_{j}^{b}=\left|\operatorname{det}\left(e_{c}^{k}\right)\right| e_{j}^{b}$ and the variant $K_{a}^{i}=e^{i b} K_{a b}$ of extrinsic curvature, or Ashtekar-Barbero variables [17, 18] $\left(A_{a}^{i}, E_{j}^{b}\right)$ with the connection $A_{a}^{i}=\Gamma_{a}^{i}+\gamma K_{a}^{i}$, using the spin connection $\Gamma_{a}^{i}$ and the real-valued Barbero-Immirzi parameter $\gamma[18,19]$. The latter are used in loop quantum gravity [20-22], which is based on a representation of holonomies of $A_{a}^{i}$. For further details on canonical gravity, see [23].

\subsection{Classical theory}

In ADM variables, we have the relation

$$
p^{a b}(x)=\frac{\sqrt{\operatorname{det} h}}{16 \pi G}\left(K^{a b}-K_{c}^{c} h^{a b}\right)
$$

between the momentum and the extrinsic curvature $K_{a b}$. The variables are subject to the Hamiltonian constraint

$$
C_{\text {grav }}[N]=\int \mathrm{d}^{3} x N\left\{\frac{16 \pi G}{\sqrt{\operatorname{det} h}}\left[p_{a b} p^{a b}-\frac{1}{2}\left(p_{a}^{a}\right)^{2}\right]-\frac{\sqrt{\operatorname{det} h}}{16 \pi G}^{(3)} R\right\},
$$

with the spatial Ricci scalar ${ }^{(3)} R$ computed from $h_{a b}$, and the diffeomorphism constraint

$$
\vec{D}_{\text {grav }}[\vec{M}]=2 \int \mathrm{d}^{3} x M^{a} \sqrt{\operatorname{det} h} D^{b}\left[(\operatorname{det} h)^{-1 / 2} p_{a b}\right]=2 \int \mathrm{d}^{3} x M^{a} D_{b} p_{a}^{b}
$$


where $D_{a}$ is the spatial covariant derivative. The full constraints are obtained by adding the matter Hamiltonian to get $C[N]=C_{\text {grav }}[N]+H_{\text {matter }}[N]$, and an energy-flux contribution from matter to get $\vec{D}[\vec{M}]=\vec{D}_{\text {grav }}[\vec{M}]+\vec{D}_{\text {matter }}[\vec{M}]$. The matter contribution to $\vec{D}[\vec{M}]$ is usually of a simple form in which momenta are multiplied with spatial derivatives of the configuration variables, such as $\int \mathrm{d}^{3} x M^{a} p_{\phi} D_{a} \phi$ for a scalar field. (For variables with density weight one, the derivative will appear on the momentum.)

For Ashtekar-Barbero variables, we have the gravitational contribution to the diffeomorphism constraint given by

$$
\vec{D}_{\text {grav }}[\vec{M}]=\int \mathrm{d}^{3} x M^{a} P_{j}^{b}\left[F_{a b}^{j}+\left(1+\gamma^{2}\right) \epsilon^{j}{ }_{k l} K_{a}^{k} K_{b}^{l}\right],
$$

with the Yang-Mills curvature $F_{a b}^{i}=2 \partial_{[a} A_{b]}^{i}+\epsilon^{i j k} A_{a}^{j} A_{b}^{k}$ of $A_{a}^{i}$, and the contribution

$$
C_{\text {grav }}[N]=4 \pi G \gamma^{2} \int \mathrm{d}^{3} x N \frac{P_{i}^{a} P_{j}^{b}}{\sqrt{\operatorname{det} h}} \epsilon^{i j}{ }_{k}\left[F_{a b}^{k}-\left(1+\gamma^{2}\right) \epsilon^{k}{ }_{m n} K_{a}^{m} K_{b}^{n}\right]
$$

to the Hamiltonian constraint. The momentum of $A_{a}^{i}$ is $P_{i}^{a}:=E_{i}^{a} /(8 \pi \gamma G)$, so that $\left\{A_{a}^{i}(x), P_{j}^{b}(y)\right\}=$ $\delta_{j}^{i} \delta_{a}^{b} \delta(x, y)$.

Stress-energy components can be computed from the matter contributions to the Hamiltonian and diffeomorphism constraint. The most immediate variables are those measured by a so-called Euclidean observer, one that moves along geodesics with 4-velocity given by the unit normals to spacelike hypersurfaces $t=$ const in (2.1): the energy density, energy current and spatial stress are (see, e.g., $[23,24])$

$$
\rho_{\mathrm{E}}=\frac{1}{\sqrt{\operatorname{det} h}} \frac{\delta C_{\text {matter }}[N]}{\delta N}, \quad J_{a}^{\mathrm{E}}=\frac{1}{\sqrt{\operatorname{det} h}} \frac{\delta \vec{D}_{\text {matter }}\left[M^{a}\right]}{\delta M^{a}}, \quad S_{a b}^{\mathrm{E}}=-\frac{2}{N \sqrt{\operatorname{det} h}} \frac{\delta C_{\text {matter }}[N]}{\delta h^{a b}} .
$$

The matter energy density measured by a generic observer with 4 -velocity $\mathbf{u}=\left(u^{0}, \vec{u}\right)$ is then

$$
\rho=\left(u^{0}\right)^{2} \rho_{\mathrm{E}}+2 u^{0} u^{a} J_{a}^{\mathrm{E}}+u^{a} u^{b} S_{a b}^{\mathrm{E}} .
$$

The expression for spatial stress $S_{a b}^{\mathrm{E}}$ contains a functional derivative with respect to the spatial metric. In order to make a connection with the loop cosmological perturbation theory, it is more convenient to rewrite it in terms of the (densitized) triad. We start with the definition

$$
h^{a b}=\frac{E_{i}^{a} E_{i}^{b}}{|\operatorname{det} E|},
$$

where $\operatorname{det} h=|\operatorname{det} E|$, and a useful auxiliary relation

$$
\delta(\operatorname{det} E)=\operatorname{det} E \underline{E}_{a}^{i} \delta E_{i}^{a}
$$

in terms of the inverse triad, given by

$$
\underline{E}_{a}^{i}=\frac{\epsilon_{a b c} \epsilon^{i j k} E_{j}^{b} E_{k}^{c}}{3 ! \operatorname{det} E}
$$

Using the above, the variation of the spatial metric can be rewritten as

$$
\delta h^{a b}=\frac{1}{|\operatorname{det} E|}\left(E_{i}^{a} \delta E_{i}^{b}+E_{i}^{b} \delta E_{i}^{a}-E_{i}^{a} E_{i}^{b} \underline{E}_{c}^{k} \delta E_{k}^{c}\right) .
$$


According to (2.7), the variation of the matter constraint associated with the variation of the spatial metric is

$$
\delta C_{\text {matter }}[N]=-\frac{1}{2} \int \mathrm{d}^{3} x N \sqrt{\operatorname{det} h} S_{a b}^{\mathrm{E}} \delta h^{a b} .
$$

Substituting (2.11) in the last expression and using the symmetry of the spatial stress tensor, $S_{a b}^{\mathrm{E}}=S_{b a}^{\mathrm{E}}$, we obtain

$$
\delta C_{\text {matter }}[N]=-\frac{1}{2} \int \mathrm{d}^{3} x \frac{N}{\sqrt{|\operatorname{det} E|}} S_{a b}^{\mathrm{E}}\left(2 E_{i}^{a} \delta E_{i}^{b}-E_{i}^{a} E_{i}^{b} \underline{E}_{c}^{k} \delta E_{k}^{c}\right) .
$$

Taking a functional derivative with respect to $E_{k}^{c}$ yields

$$
\frac{\delta C_{\mathrm{matter}}[N]}{\delta E_{k}^{c}}=-\frac{N}{2 \sqrt{|\operatorname{det} E|}}\left(2 S_{a c}^{\mathrm{E}} E_{k}^{a}-S_{a b}^{\mathrm{E}} E_{i}^{a} E_{i}^{b} \underline{E}_{c}^{k}\right) .
$$

The last term is proportional to the trace of the spatial stress tensor, $S_{a b}^{\mathrm{E}} h^{a b}$, or the pressure

$$
P_{\mathrm{E}}=\frac{1}{3} S_{a b}^{\mathrm{E}} h^{a b}=\frac{2 E_{k}^{c}}{3 N \sqrt{|\operatorname{det} E|}} \frac{\delta C_{\mathrm{matter}}[N]}{\delta E_{k}^{c}} .
$$

Substituting the trace back into (2.14), contracting with $E_{k}^{b}$, and solving for $S_{a b}^{\mathrm{E}}$, we obtain the spatial stress tensor

$$
S_{a b}^{\mathrm{E}}=-\frac{1}{N \sqrt{|\operatorname{det} E|}} h_{a c} \frac{\delta C_{\mathrm{matter}}[N]}{\delta E_{i}^{d}}\left(\delta_{b}^{d} E_{i}^{c}-\delta_{b}^{c} E_{i}^{d}\right),
$$

where $h_{a b}$ is the inverse of (2.9) as a function of the densitized triad.

As shown in [24], the local conservation law of the stress-energy tensor, which can canonically be derived from the algebra (1.1)-(1.3), no longer holds when the algebra is deformed. However, a consistent form of continuity equations for the components (2.7) remains intact. Specifically, the Poisson braket of (2.7) with the constraints should vanish weakly. If one does not have information on the off-shell algebra, no affirmative statement about energy conservation can be made.

\subsection{Quantization}

In order to quantize the theory canonically, one turns the phase-space variables into basic operators acting on wave functions of a certain kind. In terms of $\left(h_{a b}, p^{a b}\right)$, one usually employs formal constructions in which " $\hat{h}_{a b}(x)$ " is a multiplication operator on a functional $\psi\left[h_{a b}\right]$ and " $\hat{p}^{a b}(x)$ " a functional derivative. Difficulties in making sense of such tensorial operators of local fields can be overcome with a loop quantization [25]. As basic objects one introduces holonomies $h_{e}(A)=\mathcal{P} \exp \left(\int_{e} \mathrm{~d} \lambda \tau_{i} A_{a}^{i} \dot{e}^{a}\right)$ (with Pauli matrices $2 i \tau_{i}$ ) assigned to curves $e$ in space, and fluxes $F_{S}^{(f)}(E)=\int_{S} \mathrm{~d}^{2} y E_{i}^{a} f^{i}$ assigned to surfaces $S$ in space (and with $\mathrm{su}(2)$-valued smearing functions $f^{i}$ ). Holonomies and fluxes, when known for all curves and surfaces, allow one to reconstruct the local fields $A_{a}^{i}$ and $E_{j}^{b}$. Instead of being space-time tensors, they take values in the group $\mathrm{SU}(2)$ or its Lie algebra, and therefore have more manageable transformation properties. Moreover, they are integrated or smeared in such a way that a well-defined and non-distributional algebra under Poisson brackets results (free 
of $\delta$-functions). Its representation on functions of holonomies is the quantum representation used in loop quantum gravity.

It is now $h_{e}(A)$ that serves as a multiplication operator, for every curve $e$ in space. Fluxes, quantizing the densitized triad, are related to derivative operators, taking the form of angular-momentum operators on $\mathrm{SU}(2)$. They have discrete spectra, indicating that space acquires a discrete geometric structure [26, 27].

The question of what geometric structure space-time has in this theory is more complicated, and not completely solved. At this stage, the crucial issues to be faced by canonical quantizations of gravity enter. Space-time structure is determined by properties of the constraints $C$ and $\vec{D}$ that provide consistent evolution equations for the metric (or densitized triad) and its modes, especially by the algebra (1.1)-(1.3) or a quantum version of it. At the same time, they generate gauge transformations which classically take the form of coordinate changes in space-time. These constraints must be turned into operators, but they depend on the connection while only holonomies are represented in the Hilbert space of loop quantum gravity. For quantized constraints, especially $C$, one therefore modifies (or, using a common euphemism, "regularizes") the classical expression before it can be represented as an operator $[28,29]$. One uses combinations of holonomies so that for short curves $e$ (or small curvature components that enter the connection) they agree with the classical functional of connection components. The precise classical expression is obtained only in a limit, so that for any extended curve there are quantum corrections. They can be understood as a power series

$$
h_{e}(A)=1+\epsilon \tau_{i} A_{a}^{i} \dot{e}^{a}+\cdots,
$$

where $\epsilon$ is a small parameter depending on the coordinate length of the curve $e$. In addition to higher powers of the connection, there are higher spatial derivatives when the integration over $e$ is suitably expanded. (The treatment of higher spatial derivatives has several subtleties; see $[30]$.)

It turns out that there is a second type of correction in loop quantum gravity. The classical constraint $C$ depends on an inverse of the densitized triad, or on its inverse determinant. Since the densitized triad is quantized by flux operators with zero in their discrete spectra, there is no densely-defined inverse operator. Nevertheless, one can quantize the inverse in a more indirect way, making use of the classical identity [29]

$$
\left\{A_{a}^{i}, \int \mathrm{d}^{3} x \sqrt{\left|\operatorname{det} E_{j}^{b}\right|}\right\} \propto \epsilon^{i j k} \epsilon_{a b c} \frac{E_{j}^{b} E_{k}^{c}}{\sqrt{\left|\operatorname{det} E_{l}^{d}\right|}} .
$$

On the left-hand side, no inverse of the densitized triad is required, and the connection can be expressed in terms of holonomies. Once the Poisson bracket is replaced by a commutator divided by $i \hbar$, a well-defined quantization of the right-hand side is obtained. This commutator reproduces the right-hand side in the classical limit for large flux eigenvalues, but shows characteristic inverse-triad corrections [31, 32] for small values.

Holonomy modifications and inverse-triad corrections change the constraint $C$ and the dynamical equations of motion it generates. A more subtle effect is a possible change in space-time structure because $C$ generates not only equations of motion but also gauge transformations under which observables are invariant. One ends up with a consistent set of equations and observables if the algebra of constraints obtained after all modifications have been inserted remains first class. Ideally, one would like to make sure that even the underlying constraint operators obey a first-class commutator algebra, but existing results remain 
incomplete. General arguments [33] only indicate that the commutators obey a version of (1.3) if the constraint $\vec{D}$ is assumed to be solved. The right-hand side of (1.3) then vanishes, but one does not see the more-specific form of the actual commutator for $\vec{D} \neq 0$. These arguments therefore do not show complete consistency [34-36], nor do they elucidate the possible form of quantum space-time structure in the theory.

There are more-detailed calculations that have shown a consistent version of (1.3) at the operator level, so far in (2+1)-dimensional models [37-40] and in spherically symmetric models [41]. These results are promising, but it remains unclear how easy it will be to extend them to the full theory. Moreover, for phenomenological or effective equations one would have to find suitable semi-classical states, so that a consistent operator algebra, if it exists, could be translated into a consistent version of quantum-corrected cosmological perturbation equations. Both steps seem very difficult at present, and therefore it is important to test possible consistent versions and their implications with effective methods such as those described here, computing the algebra of modified classical constraints.

Also for modified classical constraints, several consistent versions have been found. The set of all these calculations (operator and effective ones) shows mutual consistency: In all cases, the classical algebra is deformed by quantum corrections in the same way, changing (1.3) to

$$
\left\{C\left[N_{1}\right], C\left[N_{2}\right]\right\}=\vec{D}\left[\beta\left(N_{1} \vec{\nabla} N_{2}-N_{2} \vec{\nabla} N_{1}\right)\right]
$$

with a phase-space function $\beta$, while the other Poisson brackets involving $\vec{D}$ remain unmodified. The form of $\beta$ depends only on what quantum correction is implemented but not on the models or methods used to derive the algebra. (See section 3.2 for more details.) No generic undeformed consistent version has been found, so that quantum corrections in the constraint algebra, especially in (1.3), appear to be a universal feature of models of loop quantum gravity.

\subsection{Quantum corrections}

Loop quantum gravity gives rise to holonomy modifications and inverse-triad corrections, and other canonical approaches provide less clearly-defined quantum terms resulting for instance from factor ordering or the regularization of functional derivatives. Since loop modifications are closely related to the basic representation and therefore very characteristic of the theory, their detailed study is of considerable interest in order to see if the theory can be falsified.

These loop corrections modify the classical constraints but do not introduce new degrees of freedom: One just quantizes the classical phase space by turning a preferred set of variables into operators. The form of the latter then requires certain adaptations of the constraints that generate the dynamics, and quantum corrections result. If momenta are related to first time derivatives, upon using equations of motion, non-standard kinetic terms may be obtained with higher powers of the first derivative (but no higher time derivatives). Nowhere in this process do new degrees of freedom or modifications of the phase space structures arise.

\subsubsection{Degrees of freedom and effective phase space}

The preceding statement may come as a surprise because holonomy corrections may look like (and are often taken* as examples of) familiar higher-curvature corrections generically

\footnotetext{
* See, for instance, the statements in [42], expanded in [43]. While these papers provide candidates for higher-curvature actions that could capture some implications of holonomy modifications, they do not deal directly with holonomy terms or higher orders in a connection expansion such as (2.17). Instead, they take
} 
expected for quantum gravity $[48,49]$. However, as already suggested by the origin of holonomy modifications, they are not of higher-curvature type: Holonomy corrections arise from considerations of spatial quantum geometry, not from possible forms of space-time covariant actions. One would, of course, expect quantum corrections to respect some kind of space-time covariance for the theory to be consistent, but in canonical approaches this is something to be shown, not to be assumed. Before covariance structures of the modified constraints are analyzed (by studying the first-class nature of their Poisson brackets), one cannot be sure that holonomy corrections are of standard higher-curvature type.

Nevertheless, holonomy modifications provide corrections that depend on certain measures of curvature. The connection is a linear combination of the spin connection (related to intrinsic curvature of space) and extrinsic curvature. Furthermore, one may, for dimensional reasons, assume length parameters for curves along which holonomies are integrated to be close to the Planck length (although this is not guaranteed). We then assume that the spatial structure of an effective theory is the same as the classical one. This assumption may, of course, be questioned, but it is weaker than assuming the classical space-time structure. And it is supported by the fact that the constraint $\vec{D}$ (or the finite transformations it generates) does not receive holonomy modifications or inverse-triad corrections in loop quantum gravity [50], while $C$ does. (A modification of the theory may lead to corrections of $\vec{D}$ [51], but this possibility has not been fully analyzed.) With this assumption, one would conclude that a combination of holonomies used to "regulate" the constraint $C$ has an expansion of the form $\sqrt{\operatorname{det} h} \sum_{n=0}^{\infty} \ell_{\mathrm{P}}^{2 n}\left(\alpha_{n}{ }^{(3)} R_{n}+\beta_{n} K_{n}\right)$ with the spatial metric $h_{a b}$ and spatial curvature invariants ${ }^{(3)} R_{n}$ (depending on spatial derivatives of $h_{a b}$ ) as well as spatial scalars $K_{n}$ formed from extrinsic curvature (that is, containing time derivatives of $h_{a b}$ on shell), both of order $n$. (For $n=0,{ }^{(3)} R_{0}$ is the 3 -dimensional Ricci scalar.) Holonomy modifications therefore provide spatial higher-curvature terms, but not necessarily space-time ones.

The previous statement is related to the question of degrees of freedom. Highercurvature terms of a space-time theory imply new degrees of freedom because they always involve higher derivatives in time. (In an initial-value formulation, not just the fields and their first time derivatives must be chosen initially, but higher derivatives as well; this is the so-called Ostrogradski problem.) Such new degrees of freedom would have to modify the phase-space structure by an extension of the classical phase space. If only higher spatial derivatives are present, however, as in expanded holonomies, no new degrees of freedom arise, and the phase space does not change. ${ }^{\dagger}$ Holonomy modifications merely amount to a redef-

as their starting point a modified Friedmann equation of the form $H^{2}=(8 \pi G / 3) \rho\left(1-\rho / \rho_{0}\right)$ with some constant $\rho_{0}$, which in a restricted set of homogeneous and isotropic models has been shown to be equivalent to an effective Friedmann equation in the presence of a holonomy modification of the left-hand side $H^{2}$ [44-46]. The $\rho^{2}$-correction can be obtained from an isotropic reduction of a suitable $F(R)$ action and could be taken as a higher-curvature theory corresponding to the isotropic holonomy-modified model. However, (i) the authors of $[42,43]$ assume (but do not show) that inhomogeneous space-time can still be described by the classical notion of covariance and (ii) the relation between holonomy modifications and the simple $\rho^{2}$-correction breaks down when one goes beyond the simplest isotropic models. Therefore, [42, 43] is not directly usable to understand the fundamental nature of space-time corresponding to holonomy-modified equations. A recent result shows that a careful consideration of isotropic models with modifications suggested by loop quantum gravity in fact indicates the need for modified space-time structures: The various versions of modified Friedmann equations suggested so far in loop quantum cosmology cannot be consistent with classical coordinate transformations $[47]$.

${ }^{\dagger}$ In some theories such as Hořava-Lifshitz gravity [52], there are higher spatial but not higher time derivatives, which nevertheless give rise to new degrees of freedom. However, these degrees of freedom arise from a restriction of space-time diffeomorphisms to foliation-preserving diffeomorphisms, or from extra terms in the 
inition of the dynamics and perhaps of the space-time structure via a deformed constraint algebra. They do not modify the phase space of an effective theory.

Like any interacting quantum theory, loop quantum gravity should generically give rise to higher time derivatives and therefore new degrees of freedom in an effective description. Indeed it does, but these corrections are not the same as holonomy modifications. At a canonical level, higher-time derivatives in an effective formulation of some quantum theory arise from coupling terms between expectation values of basic operators with fluctuations and higher moments [53-55]. Appendix A provides a brief summary of the essential details of this formalism referred to here.

It is important to note that effective systems based on quantum moments are more general than what is usually considered as a local effective action. Derivative expansions of effective equations do not necessarily exist for all quantum states, which for moment equations corresponds to an adiabatic approximation (see below). Especially the algebra of effective constraints is insensitive to the existence of such regimes or expansions. All one needs is an expansion by moments which in principle could go to rather high orders. If there is a consistent quantum system with a first-class algebra of constraint operators $\hat{C}_{I}$, it follows from the methods introduced in $[58,59]$ that there is a consistent algebra of effective constraints $\left\langle\hat{C}_{I}\right\rangle$ as well as higher-order ones of the form $\left\langle\widehat{\operatorname{pol}} \hat{C}_{I}\right\rangle$ : According to (A.1), we have $\left\{\left\langle\hat{C}_{I}\right\rangle,\left\langle\hat{C}_{J}\right\rangle\right\}=\left\langle\left[\hat{C}_{I}, \hat{C}_{J}\right]\right\rangle / i \hbar$ for all $I$ and $J$. If the constraint operators are first class, $\left\{\left\langle\hat{C}_{I}\right\rangle,\left\langle\hat{C}_{J}\right\rangle\right\}$ is therefore an expectation value of a combination of constraints, which vanishes when the effective constraints are all zero. If the effective constraints $\left\langle\widehat{p o l} \hat{C}_{I}\right\rangle$ are expanded to some order in the moments, they are first class to within this order. Requiring closed effective constraints therefore does not pose conditions in addition to what one must assume for any consistent quantum theory. A closed algebra of effective constraints must exist in all regimes, not just semi-classical or low-curvature ones; one must, however, expand to higher orders in the moments as states become more quantum.

Under certain conditions, combining a semi-classical with an adiabatic expansion, equations of motion for moments, $\mathrm{d} \Delta\left(q^{a} p^{b}\right) / \mathrm{d} t=\left\{\Delta\left(q^{a} p^{b}\right),\langle\hat{H}\rangle\right\}$, can be partially solved, upon which coupling terms in expectation-value equations take on the form of higher time derivatives [53-55]. Higher time derivatives therefore result from canonical quantum gravity, and they must obey covariance conditions because the moment terms they originate from must leave the first-class nature of the effective constraints $\langle\hat{C}\rangle$ intact. These corrections are important for any theory of quantum gravity, and they are not related to or replaced by holonomy modifications. The moments provide additional degrees of freedom, with Poisson brackets derived from (A.1) and their dynamics following from (A.3).

Considering these detailed constructions, it is clear that effective calculations do not assume what phase-space structure they refer to. Poisson brackets of all variables follow from quantum commutators via (A.1). In most cases of effective constraint algebras found so far, moment-terms are ignored to avoid their more-complicated algebra. One is then dealing with holonomy modifications without considering higher time derivatives. In section 3.1 , we will comment on how meaningful this separation of holonomy modifications and higher-curvature (or moment) corrections is. What is left of the phase space is then just the expectation values of basic operators, with Poisson brackets identical to the classical ones. This fact may give rise to the impression that the classical phase-space structure is just assumed, but it is actually implied by general effective methods together with the order of calculations.

action with free auxiliary functions on top of the metric degrees of freedom. No such auxiliary functions are suggested by holonomy modifications. 
Sometimes, following [60], one uses a partial derivation of effective equations [61, 62] as a shortcut, in which one assumes the evolving state and its moments to be of a certain form, most often Gaussian for simplicity. In [60], the method has been introduced and used in order to show that the correct classical limit is obtained for loop quantum cosmology, building on [63]. But it turned out to be less reliable for a derivation of semi-classical physics with leading-order quantum corrections: In most quantum systems, an initial Gaussian state rapidly evolves away from being Gaussian (see for instance [64] in quantum cosmology). Nevertheless, there may still be interesting features shown by such models. In order to ensure that the moments $\Delta$ kept in the system are in some sense close to an evolving state, one must find a relation $\Delta(\langle\hat{q}\rangle,\langle\hat{p}\rangle)$ between them and expectation values, so that a state peaked on $(\langle\hat{q}\rangle(t),\langle\hat{p}\rangle(t))$ as it evolves according to the Schrödinger equation changes its moments by $\Delta(t)=\Delta(\langle\hat{q}\rangle(t),\langle\hat{p}\rangle(t))$. (For comparison, in general effective equations such a relation is available only in special regimes, such as adiabatic ones. Otherwise, there are coupled but independent equations for $(\langle\hat{q}\rangle(t),\langle\hat{p}\rangle(t), \Delta(t))$.) It is then possible to write the system of $(\langle\hat{q}\rangle,\langle\hat{p}\rangle, \Delta)$ as one on a phase space of classical variables $(q, p)$, with Poisson brackets given by the pull-back of the symplectic structure on state space by the embedding $(q, p) \mapsto$ $(\langle\hat{q}\rangle,\langle\hat{p}\rangle, \Delta)$. Since moments $\Delta$ contribute non-trivially to the full symplectic structure, the Poisson brackets for $q$ and $p$ derived in this way may differ from the classical one, and therefore carry quantum corrections. But such corrections follow from one's choice of state, intermingling moments with basic expectation values; they do not arise when one considers moments as independent degrees of freedom, as one must do in general regimes. Moreover, in order to find the embedding $(q, p) \mapsto(\langle\hat{q}\rangle,\langle\hat{p}\rangle, \Delta)$ one solves some equations of motion. In the present context of non-trivial constrained systems, such an embedding, as well as the whole procedure it gives rise to, is not available before a consistent algebra has been derived. It is feasible only in homogeneous models which have just a single constraint with a trivial Abelian algebra. For non-trivial effective constraint algebras, on the other hand, equations of motion cannot be used to derive the phase-space structure. Moments must be treated as independent of expectation values, and the Poisson brackets between expectation values of basic operators do not carry quantum corrections. This result is derived from the quantum theory and not postulated.

\subsubsection{Inverse-triad corrections}

In addition to these two types of corrections, holonomy modifications and quantum backreaction which both depend on curvature parameters, we have inverse-triad corrections from loop quantum gravity. These corrections have been computed in several Abelian models [31, 65-67] (using U(1) instead of $\mathrm{SU}(2)$ ) and evaluated numerically for $\mathrm{SU}(2)$ states [68]. Their construction shows that inverse-triad corrections depend on the relation of elementary flux values (or the spatial discreteness scale) to the Planck scale. More precisely, they refer to expectation values of elementary flux operators in a quantum-gravity state. For a highly refined state, these values are small. Rather surprisingly, inverse-triad corrections disappear for large flux values, where discretization effects of spatial derivatives (and, presumably, curvature terms) would become large. A parametrization of inverse-triad corrections is then often expanded as

$$
\langle\hat{E}\rangle\left\langle\widehat{E^{-1}}\right\rangle \sim 1+c \frac{\ell_{\mathrm{P}}^{2}}{|F|}+\cdots,
$$

where $F$ is some flux or area value characteristic of the regime considered. Inverse-triad corrections are therefore important in regimes different from those where holonomy modifi- 
cations or higher-curvature terms are crucial. These corrections can easily be separated from each other, allowing simplifications in the organization of calculations.

\subsubsection{Holonomy corrections and their ambiguities}

In an unexpanded form, holonomy modifications result from matrix elements of $\mathrm{SU}(2)$ holonomies. In most reduced models that have been studied in detail, a reduction from $\mathrm{SU}(2)$ to $\mathrm{U}(1)$ comes along with the imposition of spatial symmetries $[69,70]$ or diagonalization in Bianchi models [71]. Holonomy modifications then take a form, such as

$$
h(A) \sim \delta^{-1} \sin (\delta A),
$$

of almost-periodic functions of the connection components $[60,70]$. The parameter $\delta$, representing the lattice structure, remains undetermined as long as the reduced model is not derived from a consistent full theory. It is often assumed to be of Planck size, but this assumption is not well-supported. First, $\delta$ comes from the coordinate length of a curve, which by itself does not provide any physical length scale. Secondly, if one takes elementary fluxes as an estimate of $\delta$, inverse-triad corrections are large for Planckian values, implying strong deviations from classical geometry [72]. At the present state of knowledge, it is best to keep $\delta$ as an undetermined parameter, which may perhaps be restricted by consistency conditions for a first-class algebra of constraints or by future better information about a derivation from the full theory.

In addition to the unknown magnitude of $\delta$, its possible dependence on the triad is not much restricted. A $\delta$ depending on the triad amounts to lattice refinement [73, 74]: the size of curves in a discrete state changes as a space-time region expands or contracts. In the full theory, the analog of a changing $\delta$ is the creation of new vertices or curves as a discrete state evolves, which happens in current constructions of the full dynamics. Again, it is the lack of a direct derivation from the full theory that leaves ambiguities in suitable parametrizations of holonomy modifications.

Currently, the closest to a derivation of symmetric models from the full theory is a distributional construction of symmetric states and basic operators [69]. As recent considerations have shown [75], the full quantum state space cannot be captured completely by a simple reduced $\mathrm{U}(1)$-theory. It is therefore unclear if $\mathrm{U}(1)$-matrix elements such as (2.21), even with unspecified and triad-dependent $\delta$, are sufficient to model the full expressions of the quantum constraint. The almost-periodic form may be good as a first approximation, but more generally one should try to keep the functional form of $h(A)$ as unrestricted as possible. As shown especially by spherically symmetric models, summarized in section 3.2 , interesting results can still be found.

\subsubsection{Relation to homogeneous quantum cosmology (mini-superspace models)}

Before we continue with a more detailed consideration of the constraint algebra, it is useful to recall the steps undertaken to formulate homogeneous models of loop quantum cosmology. As in the full theory, one starts by modifying the classical constraint so as to make it representable by holonomies. The resulting expression is then quantized straightforwardly, just inserting holonomy and flux (or inverse-flux) operators. When choosing the modification (or the quantization if the modification is done only implicitly), one is subject to the same ambiguities, choices and unknowns as just described for inhomogeneous models, and the resulting state equations for wave functions are no less ambiguous than the effective constraints including holonomy modifications. 
After formulating the state equation, one has different options for a further analysis. One may just solve the partial differential or difference equation for the wave function, but then still has to compute expectation values of suitable observables to derive physical implications. Effective methods applied to homogeneous models provide a shortcut by which one can analyze the same state equation but without computing a complete wave function. Being based on expectation values and moments in their canonical incarnation, effective methods directly produce results for observable quantities.

In the inhomogeneous context, effective methods for constrained systems realize a further simplification, as already mentioned. For state equations, one would have to show that constraint operators obey a first-class algebra before they can consistently be solved. With the effective approach used here, consistency is checked in terms of Poisson brackets, and once it is realized, equations of motion for observables can directly be computed. In summary, effective and standard constructions of models of quantum cosmology and their solutions for wave functions are based on the same assumptions as far as their relation to some full quantum gravity is concerned, and they are subject to the same ambiguities. The difference lies in the approximations they employ to derive solutions.

\section{Constraint algebra and space-time structure}

With the three types of corrections discussed in the preceding section (moment terms, holonomy modifications, and inverse-triad corrections) we arrive at a modified constraint $C$. For consistency, it must be part of a first-class algebra with the standard $\vec{D}$, so that equations of motion and observables can be derived without conflicting statements.

\subsection{Isolated modifications}

In general, all three types of corrections should be included in a modified constraint of effective loop quantum gravity. However, we have already seen that inverse-triad corrections depend on different quantities than holonomy modifications and moment terms, and that they are in general relevant in different regimes. It is therefore meaningful to study these corrections in separation from the others, simplifying the system for a further analysis as done in [11]. These corrections may then have potential observational consequences even in regimes that are far from Planckian curvature [32, 67, 76].

When curvature becomes large, however, moment terms and holonomy modifications must be included. It is not easy to separate these types of corrections referring only to their dependence on the density or other parameters that characterize the evolution of a state. Nevertheless, it is possible to separate the computation of consistent versions with holonomy modifications on the one hand, and moment terms on the other, and still infer reliable features of space-time structure. Holonomy modifications change the dependence of the effective constraint on expectation values of the connection, compared with the classical expression. Independently of the specific form of this modification, the presence of higherorder terms and higher spatial derivatives of the connection is a general feature. Moment terms, on the other hand, introduce new quantum degrees of freedom and their couplings to expectation values. They may be derived for the standard constraint, or one already modified by holonomy expressions. In either case, the general feature is the presence of moments in addition to expectation values.

In order to derive a consistent system, we are then looking at Poisson brackets of the original classical constraints with different types of characteristic terms added to them. For 
canonical variables such as the connection (or extrinsic curvature) and the densitized triad, the Poisson bracket of a moment and an expectation value, derived using (A.1), always vanishes. Moment terms contribute to the Poisson bracket of two constraints only when at least one moment is included on both sides of the bracket. Another general consequence of (A.1) is that the Poisson bracket of two moments always produces terms containing at least one moment. Moments therefore modify the Poisson bracket of two constraints by adding moment terms to the classical (or modified) bracket. Any anomaly possibly produced by holonomy modifications will therefore remain in the system when moment terms are added; it cannot be canceled generically, owing to the different forms of new terms. (By a detailed analysis of the structure of effective constrained systems $\left\langle\widehat{\text { pol }} \hat{C}_{I}\right\rangle$ introduced in [58, 59], one can show that moment corrections do not deform the constraint algebra of the $\left\langle\hat{C}_{I}\right\rangle[77]$. This observation is consistent with the fact that higher-curvature effective actions do not modify the classical algebra (1.1)-(1.3) [78].) A system of constraints containing holonomy modifications and moment terms can be consistent only when the holonomy-modified version alone already produces a consistent version. And any potential quantum correction in the constraint algebra of holonomy-modified constraints implies a correction in the algebra that contains also moment terms. It is therefore meaningful to study holonomy modifications in isolation, even though numerically they are expected to be of similar size as moment terms or higher-curvature corrections. Several cosmological analyses have already been performed in this vein $[13,79,80]$.

In the interplay of holonomy modifications and moment terms just described, one could have cancellations only if the values of moments are tightly related to expectation values, so that moment-independent anomalies could be canceled by moment-dependent terms. In this case, the state would be restricted by sharp relations between moments and expectation values. Formally, a consistent version of constraints would result, and the restrictions on states may even be of interest in early-universe cosmology, considering possible derived initial conditions. (But one would have to make sure that the restricted states are still consistent with the correct semi-classical limit of the theory.) However, such effective constraints would not belong to a consistent quantum theory because the requirement of a first-class algebra (and not just consistency of the constraint equations themselves) would restrict the states. This situation would be similar to the one encountered when anomalous constraint operators overconstrain states by

$$
\left[\hat{O}_{1}, \hat{O}_{2}\right] \psi=\hat{O}_{1} \hat{O}_{2} \psi-\hat{O}_{2} \hat{O}_{1} \psi=0
$$

which is a condition independent of $\hat{O}_{1} \psi=0=\hat{O}_{2} \psi$ for constraint operators that are not first class.

\subsection{Examples of anomaly-free constraints}

Consistent deformations of the classical algebra have been found in two classes of models, spherically symmetric ones and cosmological perturbations. In order to compare these results, we include here brief summaries; see $[12,30,81]$ for details. 


\subsubsection{Spherical symmetry}

For spherical symmetry, a general Hamiltonian constraint can be written as

$$
\begin{gathered}
C_{\text {grav }}^{\mathrm{Q}}[N]=-\frac{1}{2 G} \int \mathrm{d} x N\left[\alpha\left|E^{x}\right|^{-\frac{1}{2}} E^{\varphi} f_{1}\left(K_{\varphi}, K_{x}\right)+2 \bar{\alpha}\left|E^{x}\right|^{\frac{1}{2}} f_{2}\left(K_{\varphi}, K_{x}\right)\right. \\
\left.+\alpha_{\Gamma}\left|E^{x}\right|^{-\frac{1}{2}}\left(1-\Gamma_{\varphi}^{2}\right) E^{\varphi}+2 \bar{\alpha}_{\Gamma} \Gamma_{\varphi}^{\prime}\left|E^{x}\right|^{\frac{1}{2}}\right],
\end{gathered}
$$

a function of two pairs of canonical fields $\left(K_{x}, E^{x}\right)$ and $\left(K_{\varphi}, E^{\varphi}\right)$ where $\Gamma_{\varphi}=-\left(E^{x}\right)^{\prime} / 2 E^{\varphi}$. (See [82] for these spherically symmetric variables.) This form includes inverse-triad corrections if the $\alpha$ 's are not all equal to one, as well as (pointwise) holonomy corrections via two new functions $f_{1}$ and $f_{2}$. Holonomy corrections should also give rise to higher spatial derivatives if the curve integration is expanded, but the status of such terms regarding consistent constraint algebras remains incomplete [30]. Classically, $f_{1}=K_{\varphi}^{2}$ and $f_{2}=K_{x} K_{\varphi}$, as a reduction of (2.6) shows.

Anomaly freedom can be realized if $f_{2}=K_{x} F_{2}\left(K_{\varphi}, E^{x}, E^{\varphi}\right)$ provided that [12]

$$
\left[\bar{\alpha} \alpha_{\Gamma}-2 E^{x}\left(\frac{\partial \bar{\alpha}}{\partial E^{x}} \bar{\alpha}_{\Gamma}-\bar{\alpha} \frac{\partial \bar{\alpha}_{\Gamma}}{\partial E^{x}}\right)\right] F_{2}+2 \bar{\alpha} \bar{\alpha}_{\Gamma} E^{x} \frac{\partial F_{2}}{\partial E^{x}}=\frac{1}{2} \alpha \bar{\alpha}_{\Gamma} \frac{\partial f_{1}}{\partial K_{\varphi}} .
$$

(Since $f_{2}$ must depend linearly on $K_{x}$, no holonomy modifications for $K_{x}$ can be implemented in this way, unless perhaps one includes higher spatial derivatives.) The deformation function in $(2.19)$ is then

$$
\beta_{\mathrm{sph}}=\bar{\alpha} \bar{\alpha}_{\Gamma} \frac{\partial F_{2}}{\partial K_{\varphi}} .
$$

The holonomy-modification function $F_{2}$ primarily depends on $K_{\varphi}$, but it may depend on the triad components as well through lattice refinement. However, if $F_{2}$ is almost periodic in $\delta\left(E^{x}, E^{\varphi}\right) K_{\varphi}$ as expected for Abelian holonomies, $\partial F_{2} / \partial E^{x}$ is not almost periodic in $K_{\varphi}$, and (3.2) is not compatible with an almost periodic $f_{1}$. Lattice refinement can therefore not be implemented in these models, and $F_{2}$ cannot depend on $E^{x}$ if one insists on almost-periodic holonomy modifications. (It is interesting to compare this conclusion with the results of [83], a construction of homogeneous non-Abelian models which naturally lead to lattice refinement but are not based on almost-periodic functions.) With this condition, we can solve (3.2) for

$$
F_{2}=\frac{1}{2} \frac{\partial f_{1}}{\partial K_{\varphi}} \frac{\alpha \bar{\alpha}_{\Gamma}}{\bar{\alpha} \alpha_{\Gamma}-2 E^{x}\left(\bar{\alpha}_{\Gamma} \partial \bar{\alpha} / \partial E^{x}-\bar{\alpha} \partial \bar{\alpha}_{\Gamma} / \partial E^{x}\right)} .
$$

Since inverse-triad correction functions do depend on $E^{x}$, the $E^{x}$-independence of $F_{2}$ implies that

$$
\alpha \bar{\alpha}_{\Gamma}-\bar{\alpha} \alpha_{\Gamma}+2 E^{x}\left(\bar{\alpha}_{\Gamma} \frac{\partial \bar{\alpha}}{\partial E^{x}}-\bar{\alpha} \frac{\partial \bar{\alpha}_{\Gamma}}{\partial E^{x}}\right)=0
$$

and $F_{2}$ simplifies to

$$
F_{2}=\frac{1}{2} \frac{\partial f_{1}}{\partial K_{\varphi}} .
$$

(The same relation can be found using operator methods [41].) The deformation function is then

$$
\beta_{\mathrm{sph}}=\frac{1}{2} \bar{\alpha} \bar{\alpha}_{\Gamma} \frac{\partial^{2} f_{1}}{\partial K_{\varphi}^{2}} .
$$




\subsubsection{Cosmological perturbations}

For perturbative treatments, one writes the variables as $K_{a}^{i}=\bar{k} \delta_{a}^{i}+\delta K_{a}^{i}$ and $E_{i}^{a}=\bar{p} \delta_{i}^{a}+$ $\delta E_{i}^{a}$. (See section 3.3 below for further details on the perturbation scheme.) The expanded Hamiltonian constraint can then be parametrized as

$$
C_{\text {grav }}^{\mathrm{Q}}[N]=\frac{1}{8 \pi G} \int_{\Sigma} \mathrm{d}^{3} x\left[\bar{N}\left(\mathcal{H}^{(0)}+\mathcal{H}^{(2)}\right)+\delta N \mathcal{H}^{(1)}\right]
$$

where

$$
\mathcal{H}^{(0)}=-6 \gamma_{0} \sqrt{\bar{p}}(\mathbb{K}[1])^{2}
$$

contains pointwise holonomy corrections assumed to be of the specific form $\mathbb{K}[1]=\delta^{-1} \sin (\delta \bar{k})$ and inverse-triad corrections via a function $\gamma_{0}$. The first- and second-order contributions

$$
\mathcal{H}^{(1)}=-4 \gamma_{0} \sqrt{\bar{p}}\left(\mathbb{K}\left[s_{1}\right]+\alpha_{1}\right) \delta_{j}^{c} \delta K_{c}^{j}-\frac{\gamma_{0}}{\sqrt{\bar{p}}}\left(\mathbb{K}[1]^{2}+\alpha_{2}\right) \delta_{c}^{j} \delta E_{j}^{c}+\frac{2 \gamma_{0}}{\sqrt{\bar{p}}}\left(1+\alpha_{3}\right) \partial_{c} \partial^{j} \delta E_{j}^{c}
$$

with $\mathbb{K}[s]=(s \delta)^{-1} \sin (s \delta \bar{k})$, and

$$
\begin{aligned}
\mathcal{H}^{(2)}= & \gamma_{0} \sqrt{\bar{p}}\left(1+\alpha_{4}\right) \delta K_{c}^{j} \delta K_{d}^{k} \delta_{k}^{c} \delta_{j}^{d}-\gamma_{0} \sqrt{\bar{p}}\left(1+\alpha_{5}\right)\left(\delta K_{c}^{j} \delta_{j}^{c}\right)^{2} \\
& -\frac{2 \gamma_{0}}{\sqrt{\bar{p}}}\left(\mathbb{K}\left[s_{2}\right]+\alpha_{6}\right) \delta E_{j}^{c} \delta K_{c}^{j}-\frac{\gamma_{0}}{2 \bar{p}^{3 / 2}}\left(\mathbb{K}[1]^{2}+\alpha_{7}\right) \delta E_{j}^{c} \delta E_{k}^{d} \delta_{c}^{k} \delta_{d}^{j} \\
& +\frac{\gamma_{0}}{4 \bar{p}^{3 / 2}}\left(\mathbb{K}[1]^{2}+\alpha_{8}\right)\left(\delta E_{j}^{c} \delta_{c}^{j}\right)^{2}-\frac{\gamma_{0}}{2 \bar{p}^{3 / 2}}\left(1+\alpha_{9}\right) \delta^{j k}\left(\partial_{c} \delta E_{j}^{c}\right)\left(\partial_{d} \delta E_{k}^{d}\right)
\end{aligned}
$$

contain the same functions but also "counterterms" $\alpha_{i}$ inserted for sufficient freedom to incorporate consistent modifications. Also here, terms with higher spatial derivatives are expected but have not been implemented yet. Anomaly-free versions $[13,81]$ impose several conditions on the counterterms but leave $\gamma_{0}, \alpha_{3}$ and $\mathbb{K}[1]$ free. (The form $\mathbb{K}[1]=\delta^{-1} \sin (\delta \bar{k}$ ) quoted above has been chosen to agree with suggestions from mini-superspace models, but it does not follow from the condition of anomaly-freedom.) They lead to a deformation function

$$
\beta_{\text {cosmo }}=\frac{1}{2} \gamma_{0}\left(1+\alpha_{3}\right) \frac{\partial^{2}\left(\gamma_{0} \mathbb{K}[1]^{2}\right)}{\partial \bar{k}^{2}}
$$

for pure gravity. A matter Hamiltonian would have its own correction functions that can contribute factors to $\beta_{\text {cosmo }}$.

An explicit example of anomaly cancellation in the presence of inverse-volume corrections is reported in Appendix B.

\subsubsection{Comparison}

At first sight, the treatments in those two classes of models look rather different, owing to the presence of counterterms only in the perturbative approach. Existing treatments of spherically symmetric and cosmological models have indeed appeared to be rather separate from each other. However, the final results exhibit nice qualitative agreements. Our detailed comparison of the relevant terms allows us to develop a unified picture of anomaly-free constraints, which we do in the remainder of this subsection. One of the consequences is that the "counterterms" used in cosmological models, sometimes with a flavor of arbitrariness, are nothing but different parametrizations of quantization ambiguities as they also appear in spherically-symmetric models. 
As far as the inverse-triad contributions are concerned, the two factors $\bar{\alpha} \bar{\alpha}_{\Gamma}$ in (3.7) come from one term in (3.1) quadratic in extrinsic curvature and one term with second-order spatial derivatives of the densitized triad. The two factors of $\gamma_{0}$ and $\gamma_{0}\left(1+\alpha_{3}\right)$ in (3.12) come from similar terms in the perturbed Hamiltonian constraint, the term quadratic in extrinsic curvature in (3.9) and a term with second-order spatial derivatives of the densitized triad in (3.10), respectively. Even though the models as well as the calculations that lead up to a consistent constraint algebra are rather different, the forms of their deformation functions $\beta_{\mathrm{sph}}$ in (3.3) and $\beta_{\mathrm{cosmo}}$ in (3.12) are the same. For instance, for the common choice of almost-periodic holonomy modifications, such that $f_{1}\left(K_{\varphi}\right)=\delta^{-2} \sin ^{2}\left(\delta K_{\varphi}\right)$ and $\mathbb{K}[1]^{2}=\delta^{-2} \sin ^{2}(\delta \bar{k})$, we have $\beta_{\mathrm{sph}}=\cos \left(2 \delta K_{\varphi}\right)$ and $\beta_{\text {cosmo }}=\cos (2 \delta \bar{k})$ in the absence of inverse-triad corrections. These correction functions are negative at around the maximum of holonomy modifications, an interesting feature that implies signature change; see section 3.4.4.

As is clear from these comparisons, the counterterms in the perturbative treatment play a role just like the different correction functions in the parametrization for spherically symmetric models. This observation suggests a revised interpretation of counterterms. In [11], counterterms were introduced as modifications of the classical constraint that appear to be necessary for an anomaly-free algebra but, unlike primary correction functions $\gamma_{0}$, could not be guessed easily from known features of inverse-triad or holonomy operators. Moreover, the classical compact form of the full Hamiltonian constraint, in which inverse triads appear in a single factor of $\epsilon^{i j k} E_{i}^{a} E_{j}^{b} / \sqrt{|\operatorname{det} E|}$, suggested that there should be just one total correction factor for the Hamiltonian constraint in the presence of inverse-triad corrections. Since such a single factor did not lead to an anomaly-free constraint, the $\alpha_{i}$ were therefore introduced to "counter" anomalies that a uniform factor of $\gamma_{0}$ would imply.

As shown in spherically symmetric models with their four types of $\alpha$, it is more straightforward, and perhaps less confusing, to view all modified terms on the same footing. They would all come from inverse-triad corrections or from a combination with holonomy corrections, but different terms in the Hamiltonian constraint would require different modifications. The presence of several different terms merely shows that consistent modifications are not as simple as initially expected, and the freedom in inserting them in the constraint reflects general quantization ambiguities. Lacking a derivation of effective constraints from a full operator (as well as consistent versions of full operators), the form of modification functions cannot be determined without further input. Fortunately, however, the requirement of anomaly freedom implies conditions strong enough to fix some (but not all) of the free functions. (There are two types of conditions for anomaly freedom: those coming from the closure of $\{C[N], \vec{D}[\vec{M}]\}$ and those from $\left\{C\left[N_{1}\right], C\left[N_{2}\right]\right\}$. The first condition constitutes simple geometrical consistency of spatial tensors: for instance, the correction functions in $C[N]$ should yield the appropriate density weight, and all indices should be properly contracted. The latter condition, referring to space-time geometry, is much more non-trivial to satisfy and can help to rule out some quantization ambiguities.)

\subsubsection{Free functions in parametrizations}

After imposing anomaly freedom, several functions remain free. In spherically symmetric models, one of the two holonomy modification functions, $F_{2}$, is fully determined in terms of the other correction functions. Moreover, the form of these relations indicates further restrictions. For instance, because holonomy modification functions $F_{1}$ and $F_{2}$ should have a characteristic form rather different from the inverse-triad correction functions $\alpha$ and $\alpha_{\Gamma}$, 
as discussed, (3.4) implies (3.5). Only three of the inverse-triad correction functions then remain free. (One can think of this freedom as independent quantization ambiguities in the quantization of the explicit $\left|E^{x}\right|^{-1 / 2}$ in (3.1) as well as different functions of inverse triad components $1 / E^{\varphi}$ in the spin connection $\Gamma_{\varphi}$.)

\subsection{Perturbation scheme}

In cosmological models, one does not perform calculations of consistent constraint algebras for the fields $E_{i}^{a}$ and $A_{a}^{i}$ (or $K_{a}^{i}$ ), but for variables split into background quantities and inhomogeneous perturbations. This procedure is no different from some other calculations in cosmology, especially regarding the early universe. But, again following $[11,84]$ (see also $[4,5])$, there are several special constructions required for the canonical methods used to derive constraint algebras.

Assuming some inhomogeneous field $q(x)$, we split it into a background part $\bar{q}$ and its inhomogeneity $\delta q(x)$ by writing $q(x)=\bar{q}+\delta q(x) .^{\ddagger}$ For most calculations, $\delta q$ will be assumed small in cosmology, but for now the splitting is just a different parametrization of field degrees of freedom. We only require $\int \mathrm{d}^{3} x \delta q(x)=0$ in order to avoid overcounting of the homogeneous variables. In other words, $\delta q$ is a pure inhomogeneity.

If we start with general canonical variables $(q, p)$, the decompositions $q=\bar{q}+\delta q$ and $p=\bar{p}+\delta p$ map them into a larger phase space $\overline{\mathcal{P}}$ with variables $(\bar{q}, \bar{p} ; \delta q, \delta p)$. The map is not surjective unless one requires $\int \mathrm{d}^{3} x \delta q(x)=0$ and $\int \mathrm{d}^{3} x \delta p(x)=0$ on $\overline{\mathcal{P}}$, which can be done by constraints. Before these linear constraints are imposed, we have the standard Poisson brackets $\{\bar{q}, \bar{p}\}_{\overline{\mathcal{P}}}=1 / V_{0}$ and $\{\delta q(x), \delta p(y)\}_{\overline{\mathcal{P}}}=\delta(x, y)$ for two independent sets of variables, $(\bar{q}, \bar{p})$ for the background and fields $(\delta q, \delta p)$.

In order to avoid overcounting degrees of freedom, the phase space $\overline{\mathcal{P}}$ must be constrained by imposing $C_{q}=\int \mathrm{d}^{3} x \delta q(x)=0$ and $C_{p}=\int \mathrm{d}^{3} x \delta p(x)=0$. If these integrations are performed over a finite but sufficiently large region of coordinate volume $V_{0}=\int \mathrm{d}^{3} x$, we have $\left\{C_{q}, C_{p}\right\}_{\overline{\mathcal{P}}}=V_{0}$. The constraints are therefore second-class constraints, and they ensure that $\bar{q}$ and $\bar{p}$ are indeed spatial averages: $\bar{q}=V_{0}^{-1} \int \mathrm{d}^{3} x q(x)$ and $\bar{p}=V_{0}^{-1} \int \mathrm{d}^{3} x p(x)$.

The canonical structure of $q$ and $p$ follows from the Liouville term $\int \mathrm{d}^{3} x \dot{q} p$ in the canonical action. By inserting our decompositions (or pulling back the symplectic form), we find

$$
\int \mathrm{d}^{3} x \dot{q} p=\int \mathrm{d}^{3} x(\dot{\bar{q}}+\delta \dot{q})(\bar{p}+\delta p)=V_{0} \dot{\bar{q}} \bar{p}+\int \mathrm{d}^{3} x \delta \dot{q} \delta p,
$$

using the constraints $C_{q}=0$ and $C_{p}=0$. If we work on the unrestricted phase space $\overline{\mathcal{P}}$ on which $C_{q}=0$ and $C_{p}=0$ are not solved explicitly, the presence of second-class constraints is taken into account by using the Dirac bracket

$$
\{\delta q(x), \delta p(y)\}=\delta(x, y)-\frac{1}{V_{0}} .
$$

With the subtraction of $1 / V_{0}$, integrating over space on both sides, in either $x$ or $y$, produces zero. For the background variables, we have the homogeneous Poisson bracket

$$
\{\bar{q}, \bar{p}\}=\frac{1}{V_{0}} .
$$

\footnotetext{
${ }^{\ddagger}$ So far, the background for perturbative calculations in models of loop quantum cosmology has been isotropic and spatially flat. Our considerations apply also to general homogeneous backgrounds, as they would be required for anisotropic back-reaction.
} 
We now assume the inhomogeneity to be small and derive equations of motion. For equations of first order in inhomogeneity, we expand the constraints generating evolution to second order:

$$
C[N]=\int \mathrm{d}^{3} x N C=\int \mathrm{d}^{3} x\left(\bar{N} \bar{C}+\delta N C^{(1)}+\bar{N} C^{(2)}\right)
$$

(plus contributions from the diffeomorphism constraint). The superscript indicates the order in inhomogeneity, and we have used a decomposition $N=\bar{N}+\delta N$ as in the canonical variables. Taking Poisson brackets with $\delta q$, for instance, produces a first-order term from $C^{(2)}$, which thus contributes to first-order equations of motion.

In more detail, we have four types of equations: constraints

$$
\bar{C}+C^{(2)}=0 \text { and } C^{(1)}=0
$$

from varying by $\bar{N}$ and $\delta N$, and equations of motion

$$
\begin{array}{rlrl}
\dot{\bar{q}}=\left\{\bar{q}, \bar{C}[\bar{N}]+C^{(1)}[\delta N]+C^{(2)}[\bar{N}]\right\}, & & \dot{\bar{p}}=\left\{\bar{p}, \bar{C}[\bar{N}]+C^{(1)}[\delta N]+C^{(2)}[\bar{N}]\right\}, \\
& \delta \dot{q}=\left\{\delta q, C^{(1)}[\delta N]+C^{(2)}[\bar{N}]\right\}, & & \delta \dot{p}=\left\{\delta p, C^{(1)}[\delta N]+C^{(2)}[\bar{N}]\right\}
\end{array}
$$

with $\bar{C}[\bar{N}]=V_{0} \bar{N} \bar{C}, C^{(2)}[\bar{N}]=\int \mathrm{d}^{3} x \bar{N} C^{(2)}$, and so on. In perturbative treatments, the background lapse function $\bar{N}$ is usually fixed. In this way, a clear interpretation of evolution for modes on a given background results. Fixing $\bar{N}$ thereby determines the background time.

The backreaction term $b:=\left\{\bar{q}, C^{(1)}[\delta N]+C^{(2)}[\bar{N}]\right\}$ in the background equation of motion does contain $\delta N$, but it is independent of the gauge choice. To see this, we note that the modes themselves can be combined to gauge-invariant variables $\phi$ by ensuring that $\left\{\phi, C^{(1)}[\epsilon]\right\}=0$ for all $\delta N=\epsilon$ (while $\dot{\phi}=\left\{\phi, \bar{C}[\bar{N}]+C^{(2)}[\bar{N}]\right\} \neq 0$ for evolving quantities if the background gauge is fixed). For the back-reaction term, we then have $\left\{b, C^{(1)}[\epsilon]\right\}=0$ if the constraints are first class so that $\{C[\bar{N}+\delta N], C[0+\epsilon]\}=0$ when the field equations are satisfied. (We use the Jacobi identity in this statement, as well as the fact that the background term $\{\bar{q}, \bar{C}[\bar{N}]\}$ Poisson commutes with $C^{(1)}[\epsilon]$.) The back-reaction term is therefore a combination of gauge-invariant perturbations, provided the constraint algebra is first class.

In these equations, the second-order constraint is required to generate first-order equations for the modes, but it also contributes back-reaction to the background variables in (3.18). Usually, this term is small for early-universe cosmology and can be ignored when equations of motion are solved. (It cannot be ignored at the stage of constructing a consistent gauge system, for without $C^{(2)}$ in (3.18), but still in (3.19), the set of equations is not Hamiltonian, making it much more difficult to check whether the system is anomaly-free.) However, there are situations, especially derivations of non-Gaussianity, where one must go to higher orders. The perturbed equations of motion are then non-linear even for small $\delta q$, and may be difficult to solve. An alternative perturbation scheme is used in this context, in which the variables are expanded (not decomposed) as $q=q_{\mathrm{B}}+\delta^{[1]} q+\delta^{[2]} q+\cdots$, where $q_{\mathrm{B}}$ is a background zero-order quantity. To each order, linear equations of motion result if lower-order solutions have already been obtained. Such an expansion is therefore more convenient for solving the equations, but it does not give rise to a well-defined symplectic structure for the independent orders (the $\delta^{[n]} q$ are not independent degrees of freedom for different $n$ ). Therefore, it can be used only at the level of equations of motion, but not when consistent algebras and Poisson brackets are still to be computed. For the latter, the well-defined symplectic structure of $(\bar{q}, \bar{p} ; \delta q, \delta p)$ is essential.

The relation between these two types of expansions is not straightforward. Up to linear order, we have $\bar{q}=q_{\mathrm{B}}$ and $\delta q=\delta^{[1]} q$. But with higher orders, even the background variables 
do not necessarily agree because $\int \mathrm{d}^{3} x\left(\delta^{[1]} q+\delta^{[2]} q+\cdots\right) \neq 0$ in general. Moreover, $\bar{q}$ and $q_{\mathrm{B}}$ are subject to different equations, $q_{\mathrm{B}}$ to the background equation $\dot{q}_{\mathrm{B}}=\left.\{\bar{q}, \bar{C}[\bar{N}]\}\right|_{\bar{q}=q_{\mathrm{B}}}$ independent of any inhomogeneity, while the equation for $\dot{\bar{q}}$ contains a back-reaction term $\left\{\bar{q}, C^{(2)}[\bar{N}]\right\}$. In general, $\bar{q}$ and $q_{\mathrm{B}}$ are therefore different, and $\delta q \neq \delta^{[1]} q+\delta^{[2]} q+\cdots$. (The two possible schemes have been contrasted also in [85], but without paying due attention to symplectic properties and the distinction between independent degrees of freedom and higher perturbative orders.) For higher orders in inhomogeneity in situations in which a consistent constraint algebra must first be found, one uses higher-order constraints $C=$ $\int \mathrm{d}^{3} x\left[\bar{N}\left(\bar{C}+C^{(2)}+C^{(3)}\right)+\delta N\left(C^{(1)}+B^{(2)}\right)\right]$ (without $(\delta N)^{2}$ because the constraint is linear in $N$ ). As indicated, expanding the constraint $C$ now gives rise to two different second-order terms which we distinguish from each other by calling them $C^{(2)}$ and $B^{(2)}$. Here, one inserts $q=\bar{q}+\delta q$ and $p=\bar{p}+\delta p$ in the non-linear $C$ and expands in $\delta q$ and $\delta p$ to a desired order. Superscripts indicate the order in $\delta q$ taken from $q=\bar{q}+\delta q$; no $\delta^{[i]}$ is used at this stage since one still needs the symplectic structure, which only exists for $(\bar{q}, \bar{p} ; \delta q, \delta p)$, to ensure consistency and derive equations of motion and gauge transformations. If the algebra is consistent, one obtains the same type of equations as before, but with additional terms: A background constraint $\bar{C}+C^{(2)}+C^{(3)}$, which also generates background equations of motion plus back-reaction when smeared with a background lapse $\bar{N}$, a constraint $C^{(1)}+B^{(2)}$ for inhomogeneity, and their equations of motion generated by $C^{(2)}[\bar{N}]+C^{(3)}[\bar{N}]+B^{(2)}[\delta N]$.

If a consistent set of third-order constraints has been found, giving rise to second-order equations of motion, one can proceed to solving them. (No such explicit version is known yet. Second-order perturbation equations have been considered in [86], but only using conditions found for anomaly-free first-order equations.) At second order, the equations of motion for $(\bar{q}, \delta q)$ are non-linear, but they may now be reformulated for further analysis. In particular, one can, at this stage only, transform to second-order linearizing variables $\left(q_{\mathrm{B}}, \delta^{[1]} q, \delta^{[2]} q\right)$ by inserting

$$
\bar{q}=q_{\mathrm{B}}+\delta^{[1]} q+\delta^{[2]} q-\delta q
$$

in the consistent equations for $\dot{\bar{q}}+\delta \dot{q}$. (Note that $\bar{q}$ and $\delta q$ have the same evolution generator, even though some terms may drop out as in (3.19) compared to (3.18).) According to the definition of the decomposition of $q(x)=\bar{q}+\delta q$ and the expansion $q=q_{\mathrm{B}}+\delta^{[1]} q+$ $\delta^{[2]} q$, this equation, to the orders considered, is an identity. It may not be obvious to see that it provides a set of equations for $\left(q_{\mathrm{B}}, \delta^{[1]} q, \delta^{[2]} q, \ldots\right)$ only, because initially just $\bar{q}$ is eliminated while the $\delta q$ in (3.20) must still cancel those in the equation of motion. Such cancellations would be obvious if the Hamiltonian would be known to arise from one for $q(x)$, so that inserting different decompositions would just be a reformulation of variables. However, consistent constraints for cosmological perturbation theory have so far been found only at the perturbative level (and not even to third order yet) at which quantum modifications are inserted and potential anomalies canceled only after $q(x)$ has already been substituted by $\bar{q}+\delta q$. It would be extremely complicated to arrange a consistent perturbative system to one that extends to the original $q$. Nevertheless, one can see that the substitution (3.20) does lead to a set of equations for $\left(q_{\mathrm{B}}, \delta^{[1]} q, \delta^{[2]} q, \ldots\right)$. One need only note that the $\delta q$ cancel after such a substitution if the consistent perturbative system can be written as a truncation of some constraint for an undecomposed $q(x)$; for the cancellation to happen it is not necessary that this $q(x)$-system be consistent or anomaly-free. Since all higher-order terms are then completely free, such an extension easily exists and the transformation of variables is possible. The new equations are linear if one proceeds order by order in the new variables, and they are consistent to the orders considered. 


\subsection{Deformed general relativity}

While a deformation such as (2.19) provides a consistent gauge system with unambiguous physical observables decoupled from gauge degrees of freedom, it cannot belong to a spacetime theory in the classical sense. Any generally covariant theory of fields on a Riemannian space-time has as (part of) its gauge content generators that obey the classical hypersurfacedeformation algebra with (1.3), or (2.19) with $\beta= \pm 1$ (the sign being space-time signature). Gauge transformations corresponding to the algebra (1.1)-(1.3) are, on the constraint surface, in one-to-one correspondence with space-time Lie derivatives along vector fields with components determined by the smearing functions of $C$ and $\vec{D}$ [1, 87]. Second-order field equations for the metric must equal Einstein's [2], and therefore there is no room for quantum modifications, such as holonomies, that do not imply higher time derivatives. If the canonical gauge system with constraints obeying the algebra (1.1) and (1.2) with (2.19) is to be interpreted in space-time terms, one cannot refer to classical Riemannian space-time.

Such a consequence is not altogether surprising in a background-independent quantum theory of gravity. In loop quantum gravity, one takes pains avoiding any background metric structure in the definition of basic operators and the dynamics, hoping that some space-time picture emerges in a semi-classical limit. However, even if the correct semi-classical limit is realized, there is no guarantee that in any realistic regime there will be no deviations from the classical space-time structure. It is often assumed that the space-time structure remains classical while the dynamics can change, ideally in an observable way. However, the dynamics in a generally covariant theory is closely related to the space-time structure, and therefore any assumption that the latter remains unchanged is likely to be wrong. This statement is substantiated by the current constructions of consistent hypersurface-deformation algebras with modifications according to loop quantum gravity, all of which lead to deformations of the space-time structure.

\subsubsection{Deformations}

All known consistent versions of effective constraints of loop quantum gravity generically imply deformations of the algebra, with corrections not just of the constraints but also of structure functions in the form (2.19). In the models studied so far, it is possible to have modifications of the Hamiltonian constraint that respect the classical algebra, for instance for spherically symmetric inverse-triad corrections (3.1) with $\bar{\alpha}=\bar{\alpha}_{\Gamma}=1$ and $\alpha=\alpha_{\Gamma} \neq 1$ [88], or for suitable choices in cosmological models [81]. However, there is no indication why such choices should be preferred, and as they are very special, they do not give insights about the generic situation. (Moreover, if the constraint algebra is classical, it implies the classical dynamics for general, that is non-symmetric and non-perturbative, geometries [2]. The possibility of undeformed constraint algebras with modified dynamics therefore seems to be a spurious feature of the restricted situations studied so far. In fact, as shown in [14], the constraint algebra does not always uniquely determine the dynamics unless one allows for general geometries.)

The deformations found appear to be universal: For inverse-triad corrections, (1.3) is modified by the product of different types of the primary inverse-triad correction functions of the form $\alpha(\langle\hat{E}\rangle)=\langle\hat{E}\rangle\left\langle\widehat{E^{-1}}\right\rangle$. These functions are always positive and approach small values at small fluxes, where the "smallness" is determined by the discreteness scale appearing in the corrections. Such deformed algebras have been derived using effective methods for cosmological perturbations [11] and in spherically symmetric models [12, 88], as well as operator methods in $2+1$ Abelian models [38-40] and spherically symmetric models [41]. 
For holonomy corrections, the deformation (by a cosine function if the holonomy modification is by the standard sine) appears at the same place, but now depends on the connection or extrinsic curvature. In particular, this function is not restricted to be positive, implying very strong modifications at high curvature where the negative sign implies signature change [14]. More generally, as shown by (3.3) and (3.12), the deformation function $\beta$ is negative around the maximum of holonomy-modification functions, being proportional to the second derivative. Such consistent constraints have been derived in the same type of models, for cosmological perturbations [13] and in spherically symmetric models [12] using effective methods, and for operators in [37].

In both cases, the deformation function $\beta$ depends on spatial quantities (the kinematical phase-space variables) and may appear to be slicing dependent when interpreted in a conventional space-time picture. However, the fact that the theory is defined by a closed constraint algebra implies that it presents a fully consistent gauge system. There is no slicing dependence simply because a deformed algebra (2.19) belongs to a non-classical, non-Riemannian space-time structure in which the phase-space functions are no longer the standard spatial metric and extrinsic curvature. Thanks to anomaly freedom, for every classical gauge transformation that would lead to a new slicing, there is a quantum-corrected transformation in the deformed algebra. The correct number of degrees of freedom is realized, and all auxiliary structures (classically amounting to a space-time slicing) are removed. In physical terms, the system is to be evaluated by computing observables, rather than mathematical constructs such as slicings of space-time. (In some cases, interestingly, one can develop a standard space-time model by absorbing the deformation function $\beta$ in the inverse metric by a canonical transformation [89]. After the transformation, the constraint algebra then is the classical one and generates coordinate transformations. But one still does not obtain standard space-time geometry because the momenta of the new metric components are not related to extrinsic curvature.)

As already discussed, the deformations found for holonomy modifications are reliable even though moment terms have not yet been included. Moment terms cannot cancel these deformations, but they could conceivably modify them. However, no evidence for this exists at present. (Higher-curvature corrections with their time derivatives do not deform the constraint algebra [78].) Current calculations of the constraint algebra for holonomy modifications are incomplete for another reason: Although they take into account higher orders of the connection or extrinsic curvature, they do not include higher spatial derivatives as suggested by a derivative expansion of curve integrals in holonomies [66]. At high curvature, higher spatial derivatives are generically as important as higher powers of the connection; after all, Riemann curvature is a sum of quadratic combinations of the connection and its first-order spatial derivatives. Compared to inverse-triad corrections, the status of holonomy modifications is therefore less complete. Nevertheless, the deformations they imply appear to be reliable: Higher spatial derivatives would contribute terms whose order of derivatives is invariant under taking Poisson brackets. (One would just integrate by parts to rewrite expressions, preserving the number of spatial derivatives.) Any anomaly produced by the higher-order terms of holonomy modifications would therefore remain in the presence of higher spatial derivatives. (Note the similarity of this statement to the one about moments in section 3.1, which is not surprising since moments implement the canonical analog of higher time derivatives.)

The main question regarding higher spatial derivatives is not whether they would undo the higher-order deformation, but whether they can be consistently implemented at all. 
Attempts to do so in spherical symmetry [30], where such expansions have been formulated systematically, indicate that anomaly-free versions with higher spatial derivatives are much more complicated to achieve than higher-order versions, but of course, they may not be impossible. ${ }^{\S}$ If no consistent versions with higher spatial derivatives existed, loop quantum gravity and its premise of non-local basic holonomy operators would be ruled out.

\subsubsection{Evaluation of equations}

As with any first-class constrained system, there are different options to evaluate a consistent deformation of the classical hypersurface-deformation algebra for physical consequences: (i) One can try to find gauge-invariant variables and solve their equations of motion. (ii) One may pick a gauge of the consistent gauge system, or select an internal time and deparametrize in order to compute relational observables. The second option is usually easier to implement, but also the first one is feasible for linear perturbations. Both procedures rely on the consistency of the constraint algebra, for if there were anomalies (or if one just inserted modifications without checking for anomalies) gauge-invariant variables would not exist, and results would be unphysical because they would depend on which gauge or internal time one had selected.

Gauge fixing and deparametrization cannot be used reliably before a consistent representation or deformation of the algebra has been found, for this would prevent one from making sure that there are no anomalies. (Partial gauge fixings may be used to find hints at potential forms of consistent deformations with reduced effort [91]. However, there are no intrinsic means to check consistency.) It is unlikely that any consistent system results if one uses classical gauge transformations to set up the system but then modifies the dynamics, which for generally covariant theories is part of the gauge content. In this respect, quantum gravity differs crucially from other gauge theories in which (Gauss-like) constraints take much simpler forms unrelated to the dynamics. If gauge fixing or deparametrization are used carefully before inserting quantum modifications, a formally consistent system might be obtained. But one can ensure anomaly-freedom only by showing explicitly that results do not depend on the chosen gauge or internal time, and such a procedure is usually not feasible for the complicated systems subject to hypersurface deformations. Current models that do use gauge fixing or deparametrization in this way do not ask what would happen if one had made a different choice.

There is another subtlety tightly related to closure of the constraint algebra and the existence of gauge-invariant variables. Hamilton's equations explicitly contain the lapse function and shift vector. Since neither of the two is a phase-space variable, their gauge transformations are not readily available. On the other hand, different choices of the lapse and shift result in different definitions of both the geometrical and matter canonical variables. It is thus clear that the lapse and shift should be gauge transformed, too, for equations of motion to be consistent. In fact, one can deduce such transformations by changing the gauge of Hamilton's equations. Since on the left-hand side there is always a time derivative generated by a constraint, a change of gauge of the entire equation depends on the constraint algebra. As shown, for example, in [84], there are usually (many) more canonical equations than the number of lapse and shift components. Therefore, it is quite non-trivial to make all the equations of motion transform in a consistent way for one fixed lapse and shift transforma-

\footnotetext{
${ }^{\S}$ Alternatively, one could try to find a consistent deformed version of a discrete hypersurface-deformation algebra, building on [90]. However, even if such a system could be found with loop modifications, it would not give rise to a continuum effective theory.
} 
tion. Remarkably, this is possible owing to the closure of the constraint algebra. (See also [87] for an alternative approach to this issue, treating the lapse function and shift vector as additional canonical variables, thus expanding the phase space.)

First-class constraints, therefore, cannot be solved or otherwise eliminated before one implements modifications. Second-class constraints, on the other hand, play a different role, just as they do in the context of quantization. Such constraints are relevant when one uses reduced models to expand around or to compute consistent algebras in, such as spherically symmetric systems. The requirement that non-symmetric modes and their momenta vanish then contributes second-class constraints to the original first-class system. (It is sometimes stated that symmetric models follow from partial fixings of the diffeomorphism gauge. This is not true, for gauge fixing would not remove physical degrees of freedom, as must happen for a reduced model to be defined.) In contrast to first-class constraints, second-class constraints are most easily solved before quantization or modification. They do not generate gauge transformations, and therefore the issue of gauge dependence clearly does not appear. Moreover, when they are used for reduced systems, they do not imply anything about spacetime structure. They just remove degrees of freedom but do not change the structure of remaining ones. Midi-superspace models then still allow one to explore quantum space-time.

\subsubsection{Space-time}

At present, it is not clear whether there is a generalized space-time picture, such as a noncommutative $[92,93]$ or a fractional one [94, 95], suitable for the deformation (2.19). In some regimes, as shown in [96], the deformation produces effects comparable to doubly special relativity [97-99] with a deformed Poincaré algebra in the sense of [100]. This shortage of space-time models does not pose problems for physical evaluations of the theory, which can all be done at the canonical level once an anomaly-free set of constraints is given. Nevertheless, a space-time model would be useful in that it could provide additional intuition.

It is clear that any deformation in (2.19) would imply that standard space-time diffeomorphisms or coordinate changes are no longer gauge transformations of the effective metric: The algebra fails to correspond to Lie derivatives along space-time vector fields, even on the constraint surface. (It might, however, be possible to represent gauge transformations as Lie derivatives of redefined variables, as realized for instance in the examples of [89].) This fact implies that the underlying structure cannot be Riemannian, and indeed, any attempt to write a line element

$$
\mathrm{d} s^{2}=g_{a b} \mathrm{~d} x^{a} \mathrm{~d} x^{b}
$$

with $g_{a b}$ subject to modified gauge transformations of the deformed algebra would fail to produce an invariant. One would have to modify coordinate transformations in such a way that they, together with the modified gauge transformations of $g_{a b}$, cancel in the combination used for $\mathrm{d} s^{2}$. We emphasize that, in order to capture the quantum effects under discussion, it is not sufficient to allow for $g_{a b}$ to satisfy a quantum-corrected version of Einstein's equations. The classical metric tensor or curvature quantities computed from it are no longer covariant when the classical gauge algebra is deformed. (For this reason, curvature singularities in classical models of signature change, as recently considered in [102], do not have much to say about the regularity of our models of signature change with deformed space-time structures.) In the absence of a suitable space-time tensor calculus based on line elements (3.21), invariant quantities would have to be computed by canonical means, which can easily be done at the level of cosmological perturbations following [84]. 
Although the conclusions of $[2,3]$ no longer apply with a deformed algebra (2.19), the methods can still be used [14]. In this way, one can reconstruct a Lagrangian density $\mathcal{L}$ corresponding to the algebra, but an action is more difficult to obtain because the integration of $\mathcal{L}$ requires one to know the deformed space-time structure and a description in terms of coordinates or covariant measures. Even so, such reconstructed Lagrangian densities provide further insights. For a scalar field, for instance, covariance under the deformed algebra (2.19) implies that a second-order Lagrangian density can depend on derivatives of the field only via the combination

$$
\beta \chi-\left(\partial_{\perp} \phi\right)^{2}
$$

with the normal derivative $\partial_{\perp} \phi=N^{-1}\{\phi, C[N]\}$ and the spatial derivatives $\chi:=h^{a b}\left(\partial_{a} \phi\right)$ $\times\left(\partial_{b} \phi\right)$. (The derivation in $[3,14]$ requires that $\beta$ does not depend on the momentum of the metric. Deriving a generic derivative term for mode equations in the presence of holonomy corrections is therefore more complicated.) This modified derivative term makes it clear that the theory has a non-classical notion of covariance, but one that is fully consistent thanks to the anomaly-free deformation of the algebra.

The same modification appears in the gravitational mode equations, which can be derived from a specific consistent version of the modified constraints. For pure holonomy corrections in (3.10)-(3.11), for instance, we have $\beta(\bar{k})=\cos (2 \delta \bar{k})$ in the deformed algebra. One can then derive the correction to the Mukhanov-Sasaki equation of motion for gauge-invariant perturbations of scalar and tensor type $v_{\mathrm{S}, \mathrm{T}}[80]$. (These perturbations are invariant under the deformed gauge transformations generated by modified constraints. For such invariant variables to exist, off-shell closure of the constraint algebra is essential.) In conformal time $\eta$, this is given by

$$
v_{\mathrm{S}, \mathrm{T}}^{\prime \prime}-\beta \nabla^{2} v_{\mathrm{S}, \mathrm{T}}-\frac{z_{\mathrm{S}, \mathrm{T}}^{\prime \prime}}{z_{\mathrm{S}, \mathrm{T}}} v_{\mathrm{S}, \mathrm{T}}=0,
$$

which reduces to the classical equation when $\beta \rightarrow 1$. Here, primes are derivatives with respect to conformal time. This equation holds for both scalar and tensor perturbations, with suitable background functions $z_{\mathrm{S}, \mathrm{T}}$. (In the presence of inverse-triad corrections, scalar and tensor modes are subject to wave equations with different speeds $[32,81]$.) For scalar perturbations, the Mukhanov variables in the deformed case are given by

$$
v_{\mathrm{S}}=\sqrt{\bar{p}}\left(\delta \phi+\frac{\bar{\varphi}^{\prime}}{\mathcal{H}} \phi\right) \quad \text { and } \quad z_{\mathrm{S}}=\sqrt{\bar{p}} \frac{\bar{\varphi}^{\prime}}{\mathcal{H}}
$$

where $\mathcal{H}$ is the Hubble parameter in conformal time. For tensor modes, one obtains

$$
v_{\mathrm{T}}=\sqrt{\frac{\bar{p}}{|\beta|}} h \quad \text { and } \quad z_{\mathrm{T}}=\sqrt{\frac{\bar{p}}{|\beta|}},
$$

where $h$ represents the two degrees of freedom. Inserting (3.25) into (3.23), we obtain the following equation of motion for tensor perturbations:

$$
\left(h_{a}^{i}\right)^{\prime \prime}+\left(h_{a}^{i}\right)^{\prime}\left(2 \mathcal{H}-\frac{\beta^{\prime}}{\beta}\right)-\beta \nabla^{2} h_{a}^{i}=0 .
$$

The modified space-time picture, although not directly observable, has further physical consequences. Most importantly, since time translations are intimately related to energy 
conservation, the status of this important law is unclear when constraints of the hypersurfacedeformation algebra are modified. If the algebra remains consistent, energy conservation is still realized in the general sense of continuity equations [24], but without considering the algebra no such statement could be made.

\subsubsection{Signature change}

The derivative term (3.22) in a scalar Lagrangian density, as well as explicit derivations $[13,80]$ of equations for tensor modes $(3.23)$, shows that covariance and the form of dispersion relations are modified in a theory subject to (2.19). There is an even more dramatic consequence, owing to the fact that $\beta$ can become negative at high density in the presence of holonomy modifications [12, 13]. Equations of motion for modes then become elliptic rather than hyperbolic differential equations, and no well-posed initial-value problem exists. In particular, in anomaly-free cosmological models one cannot evolve through high-density regimes such as bounces. In terms of space-time, this change of signature can be seen from the fact that a relation (2.19) with $\beta=-1$ is obtained for 4-dimensional Euclidean space. (But note that there is no piece of classical Euclidean space if $\beta=-1$ is found only on one maximum-curvature slice. One should therefore not expect an effective Hamiltonian constraint to resemble the classical Euclidean one. Moreover, the space-time structure is strongly non-classical near the transition where $\beta=0$. If $\beta$ changes smoothly through zero, no singularities arise in the canonical background and mode equations, in contrast to classical models of signature change as in [101] or in the recent [102].)

Origin: Signature change appears to be a strong consequence of holonomy modifications, which had been overlooked until spherically symmetric inhomogeneity and cosmological perturbations were studied in an anomaly-free way. Indeed, without inhomogeneity, one cannot determine the signature because (i) one cannot see the relative sign between temporal and spatial derivatives and (ii) the relation (1.3) trivially equals zero in homogeneous models. Nevertheless, signature change is not a consequence of inhomogeneity, the latter rather being used as a test field. One can see this easily from the derivative term (3.22) or the one in (3.23) for negative $\beta$, it remains positive definite no matter how small the spatial derivatives are (unless they are exactly zero). Signature change is a consequence of the strong modification one makes if one uses holonomy terms at high density, not of a small amount of perturbative inhomogeneity added to the system.

To bring out this latter point more clearly, one may compute the magnitude of modifications that holonomy terms imply compared to the classical Friedmann equation. Instead of $H^{2}=(8 \pi G / 3) \rho$ for the classical squared Hubble parameter $H=\dot{a} / a$, in the presence of holonomy modifications we have

$$
\frac{\sin ^{2}(\ell K)}{\ell^{2}}=\frac{8 \pi G}{3} \rho,
$$

in which the sine function and the length parameter $\ell$ (which may be related to the Planck length and also includes the Barbero-Immirzi parameter) are subject to ambiguities. The argument $K$ of the sine function is related to the momentum canonically conjugate to the scale factor, and can be viewed as a modified version of the Hubble parameter which equals $H$ for $\ell \rightarrow 0$. We can therefore view both the classical equation and the modified version as resulting from functions of $K$, the forms of which are given by different versions of canonical constraints: $C_{\text {class }}(K)=K^{2}$ and $C_{\text {mod }}(K)=\ell^{-2} \sin ^{2}(\ell K)$. In order to provide a quantitative 
comparison of these functions, we compute the ratio $\left(C_{\text {class }}(K)-C_{\text {mod }}(K)\right) / C_{\text {class }}(K)$ for some characteristic values of $K$.

Maximum density is reached when the sine reaches the value $\sin (\ell K)=1$, so that $\ell K=\pi / 2$, giving for $\ell=\ell_{\mathrm{P}}$ the maximum density $\rho_{\max }=3 \rho_{\mathrm{P}} /(8 \pi)$. At this density, the relative magnitude of the modification, replacing $K^{2}$ by $\sin ^{2}(\ell K) / \ell^{2}$, is

$$
\frac{K^{2}-\sin ^{2}(\ell K) / \ell^{2}}{K^{2}}=1-\frac{\sin ^{2}(\ell K)}{\ell^{2} K^{2}}=1-\left(\frac{2}{\pi}\right)^{2} \approx 0.6 .
$$

The signature changes when $\cos (2 \ell K)=0$, or $\ell K=\pi / 4$, implying a relative magnitude of the modification given by $1-\sin ^{2}(\ell K) /(\ell K)^{2}=1-8 / \pi^{2} \approx 0.2$. In the Euclidean regime, holonomy modifications in the Friedmann equation change the classical term by a contribution between $20 \%$ and $60 \%$, which is certainly much larger than any inhomogeneity one would add in the perturbative regime. It is this background modification that, when embedded in a consistent and anomaly-free theory of inhomogeneity, implies drastic changes to the structure of space-time. As long as the background modification stays below $20 \%$, space-time is Lorentzian no matter how large the inhomogeneity is (within the perturbative regime). If the background modification exceeds $20 \%$, the signature changes even for tiny inhomogeneity.

Instability: A change of sign in front of the Laplacian of the Mukhanov-Sasaki equations is not a novelty in cosmology. The same effect occurs, for instance, in higher-order gravity where the Gauss-Bonnet curvature invariant is non-minimally coupled with a scalar field ([103] and references therein). In that case, this change of sign is simply interpreted as a classical instability of the perturbations on the FRW background affecting cosmological space-time scales. Within the same model, one can have further modifications to the Mukhanov-Sasaki equations which may introduce ghost and tachyon instabilities as well as superluminal propagation. Although all of these features are problematic and can be avoided by a restriction of the parameter space, the nature of the space-time wherein perturbation modes propagate remains purely classical and Lorentzian. (The theory is of higher-curvature type and does not lead to deformations of the constraint algebra.)

Here, however, the change in the perturbation equations is a direct consequence of the deformation of the constraint algebra of gravity and, hence, of a deformation of the classical space-time structure. The type of field equations changes from hyperbolic to elliptic for all modes simultaneously and does not depend on the physical meaning of a mode. Moreover, the manifold on which physical fields are defined no longer allows any causal structure at high curvature. This is the main reason why, in the present context, such an effect is regarded as a signature change of space-time rather than a simple perturbation instability.

In practical terms, signature change implies instabilities because the oscillating time dependence $\exp ( \pm i \omega t)$ is replaced by an exponential $\exp ( \pm \omega t)$. The growing mode leads to an instability if initial values are chosen at some fixed $t$. (A boundary-value problem fixing the field at two different values of $t$, as suitable for elliptic equations, eliminates the instability.) The significance of the instability depends on the $t$-range during which the growing mode is realized. If the $t$-range is of the order of a Planck time, for instance, only trans-Planckian modes will be affected by the instability and the problem may appear less severe. " The stability issue would be a version of the trans-Planckian problem.)

\footnotetext{
IThis question goes back to a suggestion by Jaume Garriga.
} 
One can estimate the $t$-range in the solvable background model of [46], in which a free massless scalar $\phi$ with momentum $p_{\phi}$ in a holonomy-modified spatially flat isotropic model is shown to imply a volume expectation value $\langle\hat{V}\rangle(\phi) \propto \cosh \left(\phi-\phi_{0}\right)$ with a constant $\phi_{0}$. This background dynamics implies signature change of inhomogeneous modes when the free scalar density $\rho_{\text {free }}=\frac{1}{2} p_{\phi}^{2} / V^{2}$ is between one half and the full Planck density; accordingly, $\langle\hat{V}\rangle$ changes by a factor of order one. (The momentum $p_{\phi}$ is constant for a free massless scalar.) Therefore, $\phi$ changes by adding a constant of order one. Using the equation of motion $\mathrm{d} \phi / \mathrm{d} t=p_{\phi} / V=\sqrt{2 \rho_{\text {free }}} \sim \sqrt{\rho_{\mathrm{P}}}$ in the high-curvature phase, the $\phi$-range of order one translates into a $t$-range of about a Planck time. Only trans-Planckian modes are then affected. However, if one tries to extend these arguments qualitatively to more general backgrounds [104], one can see that the general $t$-range is larger. The relationship between $\phi$ and $t$ is still given by the previous equation, where now $\mathrm{d} \phi / \mathrm{d} t=p_{\phi} / V=\sqrt{2 \rho_{\text {kin }}}$ is related to the kinetic energy density of the field. If there is a potential, or even just quantum fluctuations which one should expect in the high-curvature regime, the kinetic density would only be a small part of the total density and the $t$-range would be correspondingly larger for the same $\phi$-range. More and more modes of sub-Planckian frequencies will be affected by the instability, a problem which can be solved only by using a boundary-value problem as suitable for an elliptic differential equation.

We would like to stress that, due to the incomplete status of the theory, at present the signature change remains a possibility. It may turn out that there is a mechanism within loop quantum gravity by which signature change could be avoided after all." At present, however, this seems unlikely because the results, not just from the effective approach but also from operator computations of off-shell constraint algebras, appear to be very universal and generic.

\subsubsection{Phase space and the Hojman-Kuchaŕ-Teitelboim theorem}

It may be useful to stress that in the effective-constraints approach one does not impose the same phase-space degrees of freedom as in classical general relativity. The only important assumption made concerning this point is that loop quantum gravity allows for a sufficiently large class of semi-classical states in which the basic operators of the quantum theory have certain expectation values with small fluctuations. If this assumption was violated, the theory could not be considered as a consistent version of quantum gravity. If the assumption is satisfied and LQG therefore has a chance of being consistent, the expectation values just mentioned serve as variables which allow us to formulate a phase space for an effective theory. In a perturbative treatment as described in sections 3.2.2 and 3.3, the phase space we find is truncated due to the way perturbations are handled. To the $\hbar$-orders considered here, this phase space simply happens to coincide with the classical one.

Even if the effective phase space of the present approach and the one of general relativity match to lowest $\hbar$ order, the Hojman-Kuchař-Teitelboim theorem of [2] does not apply. The two main assumptions of [2] are that (a) the degrees of freedom are the same as in general relativity and that (b) the space-time structure (in the sense of hypersurface deformations) is classical. Then, the Hojman-Kuchar-Teitelboim theorem shows that the classical dynamics for second-order field equations is unique and given by Einstein's theory. When one considers canonical quantum gravity or its effective theories, the hypothesis that space-time remains

\footnotetext{
"Even if this were the case, one could not show it within mini-superspace models because one must have access to temporal and spatial variations. This is the reason why mini-superspace models of loop quantum cosmology (not necessarily Wheeler-DeWitt) do not seem to be completely viable at high density.
} 
classical is, of course, questionable. If assumption (b) is dropped, no uniqueness of constraint algebras can be achieved. This is the context in which we discuss more general versions.

\subsubsection{Possible observations}

There are two main paths to probe possible observational consequences of quantum gravity in general, and also of loop quantum gravity or cosmology. One is to rely on "low energy" effects but somehow compensate for their minuscule nature by looking at phenomena with a large relevant parameter of classical form, for instance huge times or distances over which those tiny effects, when integrated, can lead to measurable consequences. This idea is the basis for searches of Lorentz-invariance violations; for a review on such attempts in loop quantum gravity, see [105]. However, as neither a clear proof that Lorentz-invariance violations are indeed expected in loop quantum gravity, nor experimental evidence of such an energydependent time delay were established, we rather focus on the other main path: observational cosmology.

Cosmology involves a long time scale and is therefore partially related to the first type of phenomenology just described. However, at the same time it has the potential of allowing access to more energetic phenomena in the very early universe. Loop quantum cosmology indeed suggests strong modifications to the classical dynamics of space-time, as shown for instance by popular bounce models. To go beyond these background effects, perturbations by inhomogeneity have to be calculated. The framework reviewed in this article is compatible with a rigorous treatment of perturbations. Once the equations of motion for perturbations are known and a reasonable assumption for the vacuum state is made (section 4.1), the primordial power spectrum can be calculated. The latter can then be used as an input to evaluate the subsequent multipole spectrum $C_{l}$ which can directly be compared with observations. Then, either a MCMC or a Fisher analysis can be performed to compare the prediction with existing or forthcoming data.

As described earlier, there are two main classes of effective corrections that have been studied so far in loop quantum cosmology: holonomy and inverse-triad corrections (see $[83,106,107]$ for reviews). As usual, there are three kinds of perturbations (scalar, vector, tensor) which decouple at the first order. Of course, other corrections, especially quantum back-reaction, might play important roles. Many articles have been devoted to computing primordial spectra in this framework of deformed algebras. Most (but not all) of them deal with tensor modes [108-112]. In particular, it was shown in [113] that when the more-restricted deformation due to scalar modes is taken into account for tensor modes, the situation drastically changes. By regularizing the equation of motion, the resulting primordial spectrum was shown to be scale invariant in the IR limit, exponentially rising in the UV limit and oscillating in between. These effects are mainly due to high curvature.

Inverse-triad corrections are not directly related to curvature and could potentially be large in more-accessible regimes. From this perspective, they have been investigated in $[32,67,76]$.

Deformed general relativity not only implies modified gravitational dynamics but also a non-classical space-time structure. In addition to effects in equations of motion, there may be traces of quantum gravity in the choice of initial states for inflation. After all, the common selection of a vacuum state in the infinite past relies on quantum field theory in curved but classical space-time. With deformed space-time structures, several features usually taken for granted in quantum field theory change, for instance those of stress-energy tensors [24]. The 
treatment of states (initial or otherwise) must therefore be examined critically, as we will do in section 4 .

\subsection{Add-on inhomogeneity}

The derivation of an anomaly-free algebra is complicated and may be impossible in full generality. Several shortcuts to cosmological perturbations with loop modifications have therefore been pursued. We have already commented on the most obvious one (gauge fixing before implementing modifications) and its failure to capture a consistent space-time structure. The two other approaches discussed in this subsection are more subtle, but they both rely on deparametrization without checking for independence on one's choice of internal time.**

\subsubsection{Hybrid models}

Hybrid models [114] combine a Fock quantization for (non-perturbative) inhomogeneity with a loop-quantized and therefore modified homogeneous background. Gauge fixing is used to obtain a clear separation between background and inhomogeneity. This avenue has been evaluated in quite some detail in Gowdy models [115-117], for which several results on standard quantizations and their unitarity already exist [118-120]. For loop methods applied to Gowdy models, see [121, 122].

A hybrid approach amounts to quantum field theory on a modified background, which, unlike loop-quantized inhomogeneity, does not lead to problems with space-time covariance and anomalies. After all, covariant quantum field theory can be formulated on any curved background [123] without requiring Einstein's equations to hold. From the point of view of space-time structure, the Fock-quantized inhomogeneity of hybrid approaches plays the role of a matter field on some Riemannian space-time. Indeed, in spite of the use of gauge fixing, hybrid approaches are formally consistent, and it is even possible to compare several choices of gauge fixing and show that the resulting quantum theories are unitarily related [124].

While hybrid models provide consistent treatments of gauge systems, from the point of view of loop quantum gravity the truncation used appears to be too restrictive to study evolution of inhomogeneity through high density. When holonomy modifications are relevant for the background, for instance near a potential bounce, they should be important in mode equations as well. Fock-quantized inhomogeneity ignores the latter corrections, and therefore one can view hybrid models as models for loop quantum gravity only when modifications in the background are weak, restricting their use to low-curvature regimes in which the dynamics does not differ much from standard quantum field theory. Nevertheless, one can formally extend the evolution to high density, and this brings out one encouraging feature: Inhomogeneity appears to evolve through high density in an unstable manner [125], just as one expects when an evolution or initial-value picture is applied in a non-hyperbolic regime

${ }^{* *}$ The ambiguities regarding choices of constrained systems with the same constraint surface may seem, at first sight, to pose a similar problem to the choice of internal time in deparametrized models. Different constrained systems would be regarded as different theories which happen to have the same classical field equations. The choice of constrained system therefore amounts to a quantization ambiguity. Different choices of internal times partially correspond to quantization ambiguities too, but here also a lack of covariance (for some choices) plays a role. One can see this in homogeneous models, which have just one constraint and therefore a simple constraint hypersurface. Yet, different choices of internal times can easily lead to inequivalent quantizations. Our main point is that deparametrization does not make it easy to disentangle different kinds of ambiguities, while off-shell constraint algebras (classical, effective or quantum) enjoy a wider set of established techniques. 
(see section 3.4.4). Although the equations of hybrid models do not directly show signature change, their solutions seem to give rise to somewhat similar effects.

\subsubsection{Dressed metric}

The authors of [85] attempt to incorporate loop modifications in evolution equations for perturbative inhomogeneity, and they are careful in avoiding gauge fixing (but still use deparametrization). This approach is intended to deal consistently with quantum fields on a quantum background, building upon [126] (see also [127]). The result is a possible method to derive cosmological predictions in a "less effective" way that uses full wave functions. However, one can wonder if there is a consistent space-time structure in their framework as, instead of fixing the gauge, the authors solve classical constraints for gauge-invariant modes before they implement loop modifications and quantize the deparametrized Hamiltonian. The difference between classical gravity (even when linearized) and fields on a fixed background is that in the former setting one specifies how the fields transform under (linear) coordinate transformations. The same holds at the quantum level, and a consistent space-time structure is what sets apart a quantum-gravity model from a version of quantum-field theory on a possibly modified background. In deparametrized models, the evolution generator is a combination of some terms in the constraints. If the former is modified, it is questionable to assume that the constraints and perturbative observables are still classical. ${ }^{\dagger \dagger}$ The resulting equations may be formally consistent, but just as with gauge-fixed modifications, a corresponding space-time picture might not exist. Not so surprisingly, the authors see no hint of signature change and pose an initial state at the bounce point of background evolution, where the initial-value problem is hard to define if the inhomogeneities effectively live in a quantum version of four-dimensional Euclidean space at this point.

Although shown to be mathematically equivalent to some quantum field theory on a quantum geometry (a classical-type metric with quantum-corrected coefficients), the "dressed metric" approach [85] assumes, in some sense implicitly, a standard space-time structure. The validity of this assumption rests on an undeformed constraint algebra, whose very existence can be questioned in the presence of the holonomy modifications used: As shown by the derivation of anomaly-free modified constraints, especially in $[13,81]$, the algebra of constraints is generically deformed by quantum-geometry corrections. Current derivations are not complete, as described before in this article, but the mere possibility of deformations shows that standard space-time structures cannot just be assumed but would have to be derived. This is not done in the approach of [85].

The authors of [85] criticize derivations of anomaly-free constraint algebras, and in fact the whole underlying approach. Most of this criticism had already been addressed in the literature, although in somewhat dispersed locations. article summarize and strengthen previous statements and respond to the remaining issues raised in [85], making the case why the result of deformed algebras should be taken seriously. (We remind that the results of [2] do not underline any inconsistency in the deformed-algebra approach, as both the constraints and the algebra are simultaneously deformed in a consistent

\footnotetext{
${ }^{\dagger \dagger}$ As with gauge fixing, it is sometimes legitimate to solve classical constraints before quantization. For instance, at the classical level, the Gauss constraint may be solved explicitly to rewrite the spin connection in terms of the densitized triad. Importantly, this possibility remains intact at the effective level for the following reason: In the considered quantization schemes, the Gauss constraint does not acquire any quantum corrections, thus the expression for the spin connection is identical to its classical counterpart.

${ }^{\ddagger}$ The claim made in [85] that the anomaly-free algebra of [11] is not deformed is in fact not correct as the algebra is deformed as in (2.19), albeit with a positive $\beta$ that does not give rise to signature change.
} 
way, as discussed previously.) Moreover, although higher-time derivatives have not yet been included in current deformed versions, owing to the increased complexity of calculations, there is a clear procedure to do so within the general scheme of canonical effective equations. (The approach of [85] does not include higher time derivatives either, and it does not specify a clear method for doing so.)

The incompleteness of present anomaly-free constraint algebras and the specific way of introducing counterterms may certainly be criticized. (See section 3.2 for a discussion of the latter.) But the issue of anomaly freedom cannot simply be ignored and should in some way be faced also in approaches such as [85]. A fully consistent treatment of loop quantum gravity and its cosmological applications is clearly still missing. The progress made recently would benefit from a global understanding of the different issues and subtleties. No proposal made at this time addresses all of them.

\subsubsection{Relation to possible observations}

It is conceivable that observing the anisotropies of the B-mode cosmic microwave background may help to discriminate between the different theoretical approaches to loop quantum cosmology previously mentioned. One can compute the primordial power spectra for the "dressed metric" approach and frameworks which lead to instabilities (such as the hybrid approach or signature-change models in which one uses an initial-value problem throughout the elliptic phase; in the following, we consider this class of proposals as one approach). So far, this has been explicitly done assuming holonomy corrections only. For a proper comparison, the initial state of the inhomogeneous degrees of freedom can be chosen as the Minkowski vacuum in the infinite past of the contracting phase, since this allows one to set the same initial conditions for both approaches. (This would not be possible if the initial state was selected at the time of the bounce, for the semi-classical structure of the background space-time in deformed general relativity drastically differs from the semi-classical structure of the background used in the dressed-metric approach.) For such a choice of the initial conditions, existing calculations suggest that the predicted power spectra for tensor modes share common features but, more importantly, also exhibit possibly drastic differences, depending on the scales. For wavenumbers $k<\sqrt{8 \pi G \rho_{\max }}$ (i.e. large scale), both approaches for treating cosmological perturbations lead to a nearly scale-invariant (slightly blue-tilted) spectrum followed by oscillations. However, for smaller scales $k>\sqrt{8 \pi G \rho_{\max }}$, the deformed general relativity approach yields an exponential increase while the standard, slightly red-tilted inflationary power spectrum is recovered in the dressed-metric framework. (It should be noted that this UV behaviour of the primordial power spectrum in the dressedmetric approach is also obtained by fixing the initial state at the time of the bounce [85].) Such a difference could be clearly observed in the angular power spectrum of the polarized CMB anisotropies, showing that, at least in principle, cosmological observation may be used to potentially discriminate between the considered approaches.

It is less clear how one could distinguish results of hybrid models from those implied by deformed constraint algebras, given that the former seem to give rise to the same kind of instability. However, the cosmology of deformed constraint algebras still has to be fully analyzed, making use of a well-posed initial/boundary-value problem of mixed type. Some part of an initial state in the infinite past affects the expansion phase, but so do boundary values chosen for the elliptic regime. Without a detailed analysis, it is difficult to say if such a setup would make instabilities noticeable in power spectra. 


\section{States}

Canonical effective equations describe the evolution of a quantum state via the time dependence of expectation values of basic operators and their moments instead of wave functions. Correction terms compared to classical equations then depend via the moments on the state used, at least implicitly. A completely general state is difficult to parametrize, and therefore additional assumptions are often made to restrict possible choices. The most well-known one is the choice of a near-Gaussian state in low-energy effective actions, motivated physically by the nature of free vacuum states. In quantum cosmology, the question of states appears in two different forms, regarding the initial state of an inflaton and the quantum-gravity state of an evolving space-time background.

\subsection{Inflaton vacuum}

A standard ingredient of the inflationary scenario is the selection of initial conditions according to vacuum fluctuations of the curvature perturbation. These initial conditions may directly be combined with effective equations which describe the evolution of a state. Nevertheless, it is sometimes seen as awkward that a treatment of effective descriptions, working with quantum corrections to equations of classical type, refers to a wave function or full quantum state in some of its parts. One occasionally, and quite misleadingly, describes such a procedure as a "re-quantization" of an effective theory, which is supposed to be an approximation to a theory that was quantum to begin with. The separation between initial conditions and equations makes it clear that there is no such quantization after selecting initial conditions corresponding to some quantum state, and therefore the usual treatment in inflationary models does not pose a problem. Nevertheless, it is instructive to discuss how vacuum initial values can be derived at the level of effective equations.

At this stage, the feature of canonical effective equations describing the evolution of a quantum state characterized by its moments, as recalled in section 2.3.1, becomes important. Not only are expectation values subject to equations of motion correcting the classical dynamics, but moments of an evolving state have a dynamics of their own. Moreover, unlike the expectation values or classical variables, they are subject to quantum laws such as the uncertainty relation

$$
\Delta\left(q^{2}\right) \Delta\left(p^{2}\right)-\Delta(q p)^{2} \geq \frac{\hbar^{2}}{4}
$$

for a canonical pair. This relation, formulated directly for moments as they feature in effective equations, together with the dynamical statement of short-term stationarity of any ground state, allows one to select initial conditions for effective equations (see also [23, 128]).

We briefly recollect the relevant classical equations. Fourier modes of the curvature perturbation $v$ evolve according to the Mukhanov-Sasaki equation

$$
v_{k}^{\prime \prime}+\left(k^{2}-\frac{z^{\prime \prime}}{z}\right) v_{k}=0,
$$

where $z$ is related to the scale factor $a$. At large scales, ignoring the $k^{2}$-term, the solution must take the form $v_{k} \sim A(k) z$ with a normalization function $A(k)$ to be determined. One usually treats $v_{k}$ like a wave function and imposes conditions that amount to the Bunch-Davies vacuum in the infinite past, implying $A(k)=\exp (-i k \eta) / \sqrt{2 k}$. The power spectrum depends on $\left|v_{k}\right|^{2}=\left[z / z\left(k_{*}\right)\right]^{2} /(2 k)$, where $z\left(k_{*}\right)$ is taken at Hubble crossing. The normalization of $v_{k}$ 
is motivated by the inflationary postulate that $v$ initially have a magnitude given by vacuum fluctuations.

The same postulate allows us to derive the normalization condition from effective equations, not making use of wave functions. For very early times $(\eta \rightarrow-\infty)$, Eq. (4.2) is generated by a harmonic-oscillator Hamiltonian with "frequency" $k$ and "mass" $m=1$. Ground-state fluctuations then take the value $\left|\Delta v_{k}\right|=\sqrt{\hbar /(2 k)}$, which is taken as the value of $A(k)$. It is now important to note that one need not re-quantize the mentioned Hamiltonian in order to derive $\Delta v$. Canonical effective equations for the harmonic oscillator imply

$$
\begin{aligned}
& \frac{\mathrm{d} \Delta\left(q^{2}\right)}{\mathrm{d} t}=\frac{2}{m} \Delta(q p), \\
& \frac{\mathrm{d} \Delta(q p)}{\mathrm{d} t}=\frac{1}{m} \Delta\left(p^{2}\right)-m \omega^{2} \Delta\left(q^{2}\right), \\
& \frac{\mathrm{d} \Delta\left(p^{2}\right)}{\mathrm{d} t}=-4 m \omega^{2} \Delta(q p) .
\end{aligned}
$$

For a stationary state, all these time derivatives vanish, and therefore $\Delta(q p)=0$ and $\Delta\left(p^{2}\right)=$ $m^{2} \omega^{2} \Delta\left(q^{2}\right)$. Together with the requirement that the uncertainty relation be saturated for the harmonic (free-theory) ground state, we then have $\Delta\left(q^{2}\right)=\hbar /(2 m \omega)$, which amounts to $\left|\Delta v_{k}\right|^{2}=\hbar /(2 k)$ with the values of "mass" and "frequency" quoted above.

We used only effective equations to derive the magnitude of quantum fluctuations, applied to the harmonic oscillator to model the free vacuum of field modes. In a full derivation of canonical effective equations of quantum gravity or one of its models, moment equations accompany those for expectation values; it is not necessary to perform any additional quantization to derive such equations. In this way, initial conditions for inflationary scenarios can be derived at the effective level, even though the ground or vacuum state is not a semi-classical one. The resulting vacuum choice is not unique, since in the presence of loop quantum corrections different prescriptions can give rise to the same classical limit [32, 129]. This is not a matter of particular concern, as the uniqueness of the cosmological vacuum is a prerogative of standard general relativity and, in general scenarios where the dispersion relation or other ingredients are modified, one must make a specific choice based on some reasonable assumptions.

This derivation has an additional advantage, not just the one of avoiding the use of wave functions. In order to find the conditions of a Bunch-Davies vacuum, one must use quantum field theory in curved space-time. In deformed general relativity, for which loop quantum gravity provides examples, space-time does not have the classical structure, and it is not known how to perform standard quantum field theory on it (see [24]). The canonical effective equations used here, on the other hand, are still available, so that the correct initial conditions for vacuum fluctuations can be derived from moments. In particular, the deformation function $\beta$, or rather $\sqrt{|\beta|}$ then appears in the normalization via $\Delta v_{k}$, multiplying $k$. Instead of (4.2), one would have $v_{k}^{\prime \prime}+\left(\beta k^{2}-z^{\prime \prime} / z\right) v_{k}=0$ from (3.23). This correction of the normalization condition agrees with the WKB calculations of [129].

\subsection{Quantum-gravity state}

In models of quantum gravity, states must be selected or derived not just for matter or the inflaton but also for gravitational degrees of freedom. Unfortunately, distinguished classes of such states are much more difficult to find than for matter theories because the gravitational Hamiltonian is unbounded from below and does not allow a ground state. And even if a 
ground state existed under some additional conditions, most likely it would not provide a good choice of state in high-density regimes near the big bang. Gaussian states have often been considered in homogeneous cosmology and shown to capture the main semi-classical features of loop models provided the state remains sharply peaked at high density. In the solvable model of flat, isotropic space with a free, massless scalar, a sharply peaked state at large volume remains sharply peaked at high density, and semiclassical features are sufficient to conclude that there is a bounce. This conclusion, restricted to the solvable model, even holds for states more general than Gaussians [46, 130]. However, for non-solvable models and especially inhomogeneous space-times the choice of state is more delicate. Using a simple Gaussian or some other form of coherent or semi-classical wave function is not fully justified, in contrast with the case of the initial inflaton state.

If one does assume a Gaussian to evolve through a possible bounce, one presupposes that the state remains nearly classical even in a strong quantum regime where, for instance, holonomy modifications should be important. Therefore, one implicitly assumes that there is no strong quantum back-reaction, or no higher-curvature terms in addition to those higherorder modifications put in by holonomies. It is then not surprising that such states stay near those of simple holonomy-modified classical equations such as (3.27), which show a bounce by the bound imposed on the energy density. However, quantum back-reaction is ignored by choosing a Gaussian, and therefore it is not guaranteed that the bounces shown by (3.27) are reliable in loop quantum cosmology, even setting aside the issue of signature change.

A full understanding of the Planck regime in loop quantum gravity requires a detailed analysis of the dynamics of generic states. Such investigations are in principle possible using wave functions, but they have not been started yet. As long as these results remain lacking, one cannot be sure of the quantum nature of the big bang, not just about details but even about general questions such as whether there is a bounce. An analysis of generic states and their approach to the Planck regime would be easier to perform using the moment behavior described by canonical effective equations because the number of state parameters to be controlled would be smaller at any fixed order in the semi-classical expansion. Also this task, however, still remains to be completed. For now, therefore, the main cosmological lesson to be drawn from loop quantum gravity is the possible form of deformed space-time structures and their implications, but not any detailed scenario such as a bounce.

\section{Conclusions}

We have presented a detailed discussion of effective methods for models of canonical quantum gravity in relation to the anomaly problem. We have mainly discussed the overall coherence and consistency of the framework, but also provided new details and insights: (i) We have compared different anomaly-free models and showed their agreements, (ii) have considered higher-order perturbations in this framework, and (iii) have described an effective way of specifying an initial inflaton state.

Our results pave the way to several new developments, all of which would require more time and space than is available for this article. However, while important technical details still have to be completed in these directions, the conceptual discussions presented here have removed what had appeared to be difficult hurdles. In particular, our description of a framework for anomaly-free higher-order perturbations will be important for attempts to derive non-Gaussianity from models of loop quantum cosmology. Specific calculations would require long manipulations of Poisson brackets of parameterized constraints with, say, 
holonomy modifications. If anomaly-free constraints existed to higher orders, their derivation would follow the scheme provided here. If such constraints did not exist, the same scheme would allow one to test whether new kinds of corrections could provide anomaly-freedom. Our characterization of an effective description of initial inflaton states should be easier to implement. Here, the main new direction would be an exploration of effects from nonvacuum states. More generally, the construction of an anomaly-free perturbation dynamics will permit the extraction of the full set inflationary observables in loop quantum cosmology, thus completing extant results based on a partial or preliminary implementation of quantum corrections.

As emphasized, although a general framework to derive effective theories of canonical quantum gravity is available, it remains incompletely realized in present concrete calculations. Especially higher spatial and higher time derivatives must still be included in the existing models. No alternative procedure has yet yielded such terms for models of loop quantum gravity, and therefore it is unclear if they can be consistent at all. (If they cannot, there would be a significant mismatch between loop quantum gravity and general expectations from effective field theory, as realized in perturbative quantum gravity [48, 49].) Moreover, several features we described are lacking in such alternative approaches. We propose the effective approach sketched here as a candidate complete method to address the anomaly problem and to extract physical predictions from canonical quantum gravity.

\section{Acknowledgements}

We thank Ivan Agulló, Jaume Garriga, Guillermo Mena-Marugán and Edward Wilson-Ewing for discussions. This work was supported in part by NSF grants PHY-0748336 and PHY1307408. The work of G.C. is under a Ramón y Cajal contract.

\section{A Canonical effective theory}

A canonical formulation of effective equations can be obtained by working with expectation values and moments of states with respect to a basic set of operators. One can introduce a Poisson bracket on the space of expectation values by

$$
\{\langle\hat{A}\rangle,\langle\hat{B}\rangle\}:=\frac{\langle[\hat{A}, \hat{B}]\rangle}{i \hbar} .
$$

For basic operators, this Poisson bracket agrees with the classical one, by virtue of the standard quantization procedure. Imposing the Leibniz rule, the Poisson bracket is extended to fluctuations and moments of basic operators, providing non-classical degrees of freedom. If moments at arbitrary order are defined by

$$
\Delta\left(q^{a} p^{b}\right):=\left\langle\left[(\hat{q}-\langle\hat{q}\rangle)^{a}(\hat{p}-\langle\hat{p}\rangle)^{b}\right]_{\mathrm{symm}}\right\rangle
$$

with totally symmetric ordering, they all Poisson commute with expectation values $\langle\hat{q}\rangle$ and $\langle\hat{p}\rangle$ of basic operators, provided the latter form a canonical algebra with $[\hat{q}, \hat{p}]$ a constant. The moments are therefore truly independent phase-space degrees of freedom. (For a non-canonical algebra of basic variables, which sometimes appears in loop models when holonomies are not expanded, the phase-space structure is more complicated. However, at the perturbative level, an expansion of holonomies is required because higher powers of the connection in such an 
expansion will be mixed with moment terms contributing to higher time derivatives. The assumption of a canonical basic algebra is therefore a safe one.) For instance, two fluctuations have a Poisson bracket

$$
\begin{aligned}
\left\{(\Delta A)^{2},(\Delta B)^{2}\right\}= & \left\{\left\langle\hat{A}^{2}\right\rangle-\langle\hat{A}\rangle^{2},\left\langle\hat{B}^{2}\right\rangle-\langle\hat{B}\rangle^{2}\right\} \\
= & \frac{1}{i \hbar}\left(\left\langle\left[\hat{A}^{2}, \hat{B}^{2}\right]\right\rangle-2\langle\hat{A}\rangle\left\langle\left[\hat{A}, \hat{B}^{2}\right]\right\rangle-2\langle\hat{B}\rangle\left\langle\left[\hat{A}^{2}, \hat{B}\right]\right\rangle+4\langle\hat{A}\rangle\langle\hat{B}\rangle\langle[\hat{A}, \hat{B}]\rangle\right) \\
= & \left\{\left\langle\hat{A}^{2}\right\rangle,\left\langle\hat{B}^{2}\right\rangle\right\}-2\langle\hat{A}\rangle\left\{\langle\hat{A}\rangle,\left\langle\hat{B}^{2}\right\rangle\right\}-2\langle\hat{B}\rangle\left\{\left\langle\hat{A}^{2}\right\rangle,\langle\hat{B}\rangle\right\} \\
& +4\langle\hat{A}\rangle\langle\hat{B}\rangle\{\langle\hat{A}\rangle,\langle\hat{B}\rangle\},
\end{aligned}
$$

fully expressed in terms of (A.1).

The Heisenberg equation for an operator $\hat{O}$ is equivalent to a Hamiltonian equation for the expectation value $\langle\hat{O}\rangle$ with effective Hamiltonian $\langle\hat{H}\rangle$ :

$$
\frac{\mathrm{d}\langle\hat{O}\rangle}{\mathrm{d} t}=\frac{\langle[\hat{O}, \hat{H}]\rangle}{i \hbar}=\{\langle\hat{O}\rangle,\langle\hat{H}\rangle\}
$$

In order to write $\langle\hat{H}\rangle$ explicitly as a function of expectation values of basic operators and their moments, one may expand as

$$
\begin{aligned}
\langle\hat{H}\rangle & =\langle H[\langle\hat{q}\rangle+(\hat{q}-\langle\hat{q}\rangle),\langle\hat{p}\rangle+(\hat{p}-\langle\hat{p}\rangle)]\rangle \\
& =H(\langle\hat{q}\rangle,\langle\hat{p}\rangle)+\sum_{a+b=2}^{\infty} \frac{1}{a ! b !} \frac{\partial^{a+b} H(\langle\hat{q}\rangle,\langle\hat{p}\rangle)}{\partial\langle\hat{q}\rangle^{a} \partial\langle\hat{p}\rangle^{b}} \Delta\left(q^{a} p^{b}\right),
\end{aligned}
$$

assuming that the Hamiltonian operator $\hat{H}$ is ordered totally symmetrically. If it is not, one can relate it to a totally symmetric operator by adding terms to (A.4) that explicitly depend on $\hbar[56,57]$.

These effective methods apply to constrained systems by using $\langle\hat{C}\rangle$ (as well as $\langle\widehat{\operatorname{pol}} \hat{C}\rangle$ for operators $\widehat{\text { pol }}$ polynomial in $\hat{q}-\langle\hat{q}\rangle$ and $\hat{p}-\langle\hat{p}\rangle)$ instead of $\langle\hat{H}\rangle[58,59]$. An extension to quantum field theory is in progress, but many questions, including those studied in this article, can be addressed at a regularized level (such as states with a fixed graph in loop quantum gravity) with methods developed for a finite number of degrees of freedom.

\section{B Consistency of inverse-triad corrected equations}

To illustrate the idea that the consistency of quantum-corrected equations cannot be taken for granted and needs to be checked, we consider the following example. In [11] the cosmological equations of motion including inverse-triad corrections have been derived. Below we explicitly demonstrate that the set of background and perturbed equations is consistent. To lighten the notation, we removed all bars from background functions.

The quantum corrected background equations of motion are given by

$$
\begin{aligned}
\mathcal{H}^{2} & =\frac{8 \pi G}{3} \alpha\left[\frac{\dot{\varphi}^{2}}{2 \nu}+p V(\varphi)\right], \\
\dot{\mathcal{H}} & =\mathcal{H}^{2}\left(1+\frac{\alpha^{\prime} p}{\alpha}\right)-4 \pi G \frac{\alpha}{\nu} \dot{\varphi}^{2}\left(1-\frac{\nu^{\prime} p}{3 \nu}\right), \\
\ddot{\varphi} & +2 \mathcal{H} \dot{\varphi}\left(1-\frac{\nu^{\prime} p}{\nu}\right)+\nu p V_{, \varphi}(\varphi)=0,
\end{aligned}
$$


where, only in this appendix, the dot denotes a conformal time derivative and the prime indicates a derivative with respect to $p=a^{2}$. Classically, only two of the three equations are independent. Here we demonstrate that it is still the case with quantum corrections.

We start by recalling a convenient auxiliary relation for an arbitrary function $F$ of $p$

$$
[F(p)]^{\bullet}=2 \mathcal{H} p F^{\prime}(p) .
$$

Now take a conformal time derivative of the Friedmann equation (B.1). The resulting $\dot{\mathcal{H}}$ and $V(\varphi)$-terms can be eliminated using Eqs. (B.2) and (B.1), respectively. It is then easy to see that bringing all the terms on one side of the equation and dividing them by $(8 \pi G / 3)(\alpha / \nu) \dot{\varphi}$ yields the background Klein-Gordon equation (B.3).

Given anomaly-freedom of the constraints, it is possible to construct gauge-invariant variables and recast the equations of motion in a manifestly gauge-invariant manner. This analysis has been done for the perturbative constraints of [11], incorporating inverse-triad corrections of loop quantum gravity in a way which, to leading order, is anomaly-free. The final equations in terms of the scalar perturbations $\Phi$ and $\Psi$,

$$
\begin{aligned}
& \partial_{c}[\dot{\Psi}+\mathcal{H}(1+f) \Phi]=4 \pi G \frac{\alpha}{\nu} \dot{\varphi} \partial_{c} \delta \varphi \\
& \nabla^{2}\left(\alpha^{2} \Psi\right)-3 \mathcal{H}(1+f)[\dot{\Psi}+\mathcal{H}(1+f) \Phi]=4 \pi G \frac{\alpha}{\nu}\left(1+f_{3}\right)\left[\dot{\varphi} \delta \dot{\varphi}-\dot{\varphi}^{2}\left(1+f_{1}\right) \Phi\right. \\
&\left.+\nu p V_{, \varphi}(\varphi) \delta \varphi\right] \\
& 4 \pi G \frac{\alpha}{\nu}\left[\dot{\varphi} \delta \dot{\varphi}-p \nu V_{, \varphi}(\varphi) \delta \varphi\right]=\ddot{\Psi}+\mathcal{H}\left[2 \dot{\Psi}\left(1-\frac{\alpha^{\prime} p}{\alpha}\right)+\dot{\Phi}(1+f)\right] \\
&+ {\left[\dot{\mathcal{H}}+2 \mathcal{H}^{2}\left(1+f^{\prime} p-\frac{\alpha^{\prime} p}{\alpha}\right)\right] \Phi(1+f), } \\
& \partial_{a} \partial^{i}\left[\alpha^{2}(\Phi-\Psi(1+h))\right]=0, \\
& \delta \ddot{\varphi}+2 \mathcal{H} \delta \dot{\varphi}\left(1-\frac{\nu^{\prime} p}{\nu}-g_{1}^{\prime} p\right)-\nu \sigma\left(1-f_{3}\right) \nabla^{2} \delta \varphi+\nu p V, \varphi \varphi(\varphi) \delta \varphi \\
&+2\left[\nu p V,,_{\varphi}(\varphi)\left(1+f_{1}\right)-\mathcal{H} \dot{\varphi}\left(f_{3}^{\prime} p\right)\right] \Phi-\dot{\varphi}\left[\left(1+f_{1}\right) \dot{\Phi}+3\left(1+g_{1}\right) \dot{\Psi}\right]=0,
\end{aligned}
$$

with specific relationships between the correction functions $f, f_{i}, g_{1}, h, \alpha$ and $\nu$ (see below), are manifestly gauge invariant and reproduce the classical perturbed Einstein equation if one omits the quantum corrections. If the constraints had not been ensured to be anomaly-free, the resulting perturbation equations would couple gauge-dependent terms to the supposedly gauge-invariant curvature perturbation. Apart from gauge invariance, there is also a consistency issue which arises since, on general grounds, there are three unknown scalar functions subject to five equations. Moreover, with suitable spatial boundary conditions, Eqs. (B.5) and (B.8) can be used to eliminate two of these functions in terms of just one, say $\Psi$, which should satisfy the three remaining equations.

Another consistency check that is available classically is comparing the diffeomorphism constraint equation (B.5) and the perturbed Raychaudhuri equation (B.7). Specifically, one can remove the gradient from Eq. (B.5), again assuming suitable spatial boundary conditions, and take a conformal time derivative. Classically, the resulting equation is equivalent to Eq. (B.7). Importantly, this consistency remains true in the presence of quantum corrections: Eq. (B.5) implies

$$
\dot{\Psi}+\mathcal{H}(1+f) \Phi=4 \pi G \frac{\alpha}{\nu} \dot{\varphi} \delta \varphi,
$$


whose conformal time derivative yields

$$
\ddot{\Psi}+\dot{\mathcal{H}}(1+f) \Phi+\mathcal{H} \dot{f} \Phi+\mathcal{H}(1+f) \dot{\Phi}=4 \pi G\left(\frac{\alpha}{\nu} \dot{\varphi} \delta \varphi\right)^{\bullet} .
$$

Subtracting Eq. (B.7) and replacing the time derivative $\dot{f}$ with $f^{\prime} \dot{p} \equiv 2 \mathcal{H} f^{\prime} p$, we obtain

$$
-2 \mathcal{H}^{2} \Phi\left(1+f-\frac{\alpha^{\prime} p}{\alpha}\right)-2 \mathcal{H} \dot{\Psi}\left(1-\frac{\alpha^{\prime} p}{\alpha}\right)=4 \pi G\left[\left(\frac{\alpha}{\nu} \dot{\varphi} \delta \varphi\right)-\frac{\alpha}{\nu}\left(\dot{\varphi} \delta \dot{\varphi}-p \nu V_{, \varphi}(\varphi) \delta \varphi\right)\right] .
$$

Using Eq. (B.10), the left-hand side can be rewritten as

$$
\begin{aligned}
-2 \mathcal{H}^{2} \Phi\left(1+f-\frac{\alpha^{\prime} p}{\alpha}\right)-2 \mathcal{H} \dot{\Psi}\left(1-\frac{\alpha^{\prime} p}{\alpha}\right) & =-2 \mathcal{H}\left(1-\frac{\alpha^{\prime} p}{\alpha}\right)[\dot{\Psi}+\mathcal{H} \Phi(1+f)] \\
& =-2 \mathcal{H}\left(1-\frac{\alpha^{\prime} p}{\alpha}\right) 4 \pi G \frac{\alpha}{\nu} \dot{\varphi} \delta \varphi .
\end{aligned}
$$

After cancelling the factor of $4 \pi G$, Eq. (B.11) becomes

$$
-2 \mathcal{H}\left(1-\frac{\alpha^{\prime} p}{\alpha}\right) \frac{\alpha}{\nu} \dot{\varphi} \delta \varphi=\left(\frac{\alpha}{\nu} \dot{\varphi} \delta \varphi\right)-\frac{\alpha}{\nu}\left(\dot{\varphi} \delta \dot{\varphi}-p \nu V_{, \varphi}(\varphi) \delta \varphi\right) .
$$

It is easy to see that the terms containing the time derivative of the matter perturbation mutually cancel. Bringing all the remaining terms to one side of the equation results in

$$
\delta \varphi\left[\left(\frac{\alpha}{\nu} \dot{\varphi}\right)^{\bullet}+2 \mathscr{H} \frac{\alpha}{\nu}\left(1-\frac{\alpha^{\prime} p}{\alpha}\right) \dot{\varphi}+\alpha p V_{, \varphi}(\varphi)\right]=0 .
$$

Since $(\alpha / \nu)^{\bullet} \equiv 2(\alpha / \nu)^{\prime} \mathcal{H} p=2 \mathcal{H}(\alpha / \nu)\left(\alpha^{\prime} p / \alpha-\nu^{\prime} p / \nu\right)$, the expression inside the square brackets

$$
\frac{\alpha}{\nu}\left[\ddot{\varphi}+2 \mathscr{H} \dot{\varphi}\left(1-\frac{\nu^{\prime} p}{\nu}\right)+\nu p V_{, \varphi}(\varphi)\right]
$$

is exactly the quantum corrected background Klein-Gordon equation (B.3). Therefore Eq. (B.12), which constitutes the difference between the time derivative of (B.10) and Eq. (B.7), is identically satisfied, indicating equivalence of the diffeomorphism equation and the perturbed Raychaudhuri equation.

One more perspective on closure of the equations of motion is given by showing that the Klein-Gordon equation (B.9) is not independent. In the covariant formalism, it results from the energy conservation equation for the matter field: $\nabla_{\mu} T^{\mu \nu}=0$, the counterpart of the Bianchi identity of the gravitational sector. The latter equation is automatically satisfied by construction of the Einstein tensor. For this reason, the Klein-Gordon equation can be expressed in terms of the other equations and their derivatives. In the canonical formulation such an argument, referring to the Bianchi identity, is unfortunately not available, especially at the effective level, for it is a priori not clear what kind of action or space-time structure might correspond to the quantum corrected constraints. Nonetheless, we shall demonstrate the redundancy of the Klein-Gordon equation by an explicit derivation below. 
As we will make an extensive use of the anomaly freedom conditions presented in [11] and Appendix B of [84], it is convenient to summarize them here:

$$
\begin{aligned}
h+f-2 \frac{\alpha^{\prime} p}{\alpha} & =0, \\
2 f^{\prime} p+\frac{\alpha^{\prime} p}{\alpha} & =0, \\
g_{1}+f_{3}-f_{1} & =0, \\
f-f_{1}-\frac{\nu^{\prime} p}{3 \nu} & =0, \\
2 f_{3}^{\prime} p+3\left(f_{3}-f\right) & =0, \\
\left(f_{1}+f_{3}\right)^{\prime} p+\frac{\nu^{\prime} p}{\nu} & =0 .
\end{aligned}
$$

We start by noting that

$$
(1+f) \Phi=(1+f+h) \Psi=\left(1+2 \frac{\alpha^{\prime} p}{\alpha}\right) \Psi=\frac{2 \sqrt{p}}{\alpha}(\alpha \sqrt{p})^{\prime} \Psi=\frac{(\alpha \sqrt{p})^{\bullet}}{\alpha \sqrt{p} \mathcal{H}} \Psi
$$

where we have used (B.8) to replace $\Phi$ with $\Psi$ and (B.13). Using this result, Eq. (B.10) can be rewritten in the compact form

$$
(\alpha \sqrt{p} \Psi)^{\bullet}=4 \pi G \frac{\alpha^{2}}{\nu} \sqrt{p} \dot{\varphi} \delta \varphi .
$$

Also, using (B.10) to eliminate the second term in Eq. (B.6), the latter can be rewritten as

$$
\nabla^{2}(\alpha \sqrt{p} \Psi)=4 \pi G \frac{\sqrt{p}}{\nu}\left(1+f_{3}\right)\left[\dot{\varphi} \delta \dot{\varphi}-\dot{\varphi}^{2}\left(1+f_{1}\right) \Phi+\left(\nu p V_{, \varphi}+3 \mathcal{H} \dot{\varphi}\left(1+f-f_{3}\right)\right) \delta \varphi\right] .
$$

Eqs. (B.19) and (B.20) are analogous to the perturbed diffeomorphism and Hamiltonian constraint equations respectively. The difference $\left[(B .20)^{\bullet}-\nabla^{2}(B .19)\right]$ is equivalent to the perturbed Klein-Gordon equation. Indeed, consider

$$
\begin{aligned}
0= & \frac{1}{4 \pi G}\left[(B .20)^{\bullet}-\nabla^{2}(B .19)\right] \\
= & \left\{\frac{\sqrt{p}}{\nu}\left(1+f_{3}\right)\left[\dot{\varphi} \delta \dot{\varphi}-\dot{\varphi}^{2}\left(1+f_{1}\right) \Phi+\left(\nu p V_{, \varphi}+3 \mathcal{H} \dot{\varphi}\left(1+f-f_{3}\right)\right) \delta \varphi\right]\right\} \\
& -\frac{\alpha^{2}}{\nu} \sqrt{p} \dot{\varphi} \nabla^{2} \delta \varphi
\end{aligned}
$$

and collect similar terms on the right-hand side:

$\delta \ddot{\varphi}$-terms:

$$
\delta \ddot{\varphi}\left\{\dot{\varphi}\left(1+f_{3}\right) \frac{\sqrt{p}}{\nu}\right\}
$$

$\nabla^{2} \delta \varphi$-terms:

$$
\nabla^{2} \delta \varphi\left\{-\dot{\varphi} \frac{\alpha^{2} \sqrt{p}}{\nu}\right\}
$$




\section{$\delta \dot{\varphi}$-terms:}

$$
\begin{aligned}
& \delta \dot{\varphi}\left\{\left[\dot{\varphi}\left(1+f_{3}\right) \frac{\sqrt{p}}{\nu}\right]^{\bullet}+\frac{\sqrt{p}}{\nu}\left(1+f_{3}\right)\left[\nu p V_{, \varphi}+3 \mathcal{H} \dot{\varphi}\left(1+f-f_{3}\right)\right]\right\} \\
& =\delta \dot{\varphi}\left\{2 \mathcal{H} \dot{\varphi}\left(1+f_{3}+2 f_{3}^{\prime} p\right)\right\}
\end{aligned}
$$

where we have used the background Klein-Gordon equation (B.3) to get rid of the $\ddot{\varphi}$-term and the anomaly cancellation condition (B.17) to simplify the final expression.

\section{$\delta \varphi$-terms:}

$$
\begin{aligned}
& \delta \varphi\left\{\frac{\sqrt{p}}{\nu}\left(1+f_{3}\right)\left[\nu p V_{, \varphi}+3 \mathcal{H} \dot{\varphi}\left(1+f-f_{3}\right) \delta \varphi\right]\right\} \\
& =\delta \varphi\left\{\left[p^{3 / 2}\left(1+f_{3}\right) V_{, \varphi}\right]^{\bullet}+\left[3 \mathcal{H} \dot{\varphi}(1+f) \frac{\sqrt{p}}{\nu}\right]^{\bullet}\right\} \\
& =\delta \varphi\left\{p^{3 / 2}\left(1+f_{3}\right) V_{, \varphi \varphi} \dot{\varphi}+\mathcal{H} p^{3 / 2} V_{, \varphi}\left[3\left(1+f_{3}\right)+2 f_{3}^{\prime}\right]+3 \mathcal{H} \ddot{\varphi}(1+f) \frac{\sqrt{p}}{\nu}\right. \\
& \left.+3 \dot{\mathcal{H}} \dot{\varphi}(1+f) \frac{\sqrt{p}}{\nu}+3 \mathcal{H}^{2} \dot{\varphi} \frac{\sqrt{p}}{\nu}\left[1-\frac{2 \nu^{\prime} p}{\nu}+f+2 f^{\prime} p\right]\right\} \\
& =\delta \varphi\left\{p^{3 / 2}\left(1+f_{3}\right) V_{, \varphi \varphi} \dot{\varphi}-3 \frac{\dot{\varphi}^{2}}{\delta \varphi} \frac{\sqrt{p}}{\nu}\left(1+f_{1}\right)[\dot{\Psi}+\mathscr{H}(1+f) \Phi]\right\},
\end{aligned}
$$

where we again eliminated the $\ddot{\varphi}$-term using (B.3), substituted $\dot{\mathcal{H}}$ from the background Raychaudhuri equation (B.2) in which the $(4 \pi G \dot{\varphi})$-factor was expressed using (B.10). We also applied the anomaly freedom conditions (B.14), (B.16) and (B.17) to obtain the last line. Note that the second term contributes to the $\Phi$ - and $\dot{\Psi}$-terms below.

\section{$\Phi$-terms:}

$$
\begin{aligned}
& \Phi\left\{-\left[\frac{\sqrt{p}}{\nu}\left(1+f_{1}+f_{3}\right) \dot{\varphi}^{2}\right]^{\bullet}-3 \mathcal{H} \dot{\varphi}^{2} \frac{\sqrt{p}}{\nu}\left(1+f+f_{1}\right)\right\} \\
& =\Phi\left\{2 p^{3 / 2} \dot{\varphi}\left(1+f_{1}+f_{3}\right)-2 \mathcal{H} \dot{\varphi} \frac{\sqrt{p}}{\nu}\left(f_{3}^{\prime} p\right)\right\},
\end{aligned}
$$

where as before we have used (B.3) to substitute for $\ddot{\varphi}$ and (B.17) to simplify the final expression. Note also that the second term in the first line originates from Eq. (B.25).

$\dot{\Phi}$-terms:

$$
\dot{\Phi}\left\{-\frac{\sqrt{p}}{\nu}\left(1+f_{1}+f_{3}\right) \dot{\varphi}^{2}\right\}
$$

$\dot{\Psi}$-terms:

$$
\dot{\Psi}\left\{-3 \frac{\sqrt{p}}{\nu}\left(1+f_{1}\right) \dot{\varphi}^{2}\right\}=\dot{\Psi}\left\{-3 \frac{\sqrt{p}}{\nu}\left(1+f_{3}+g_{1}\right) \dot{\varphi}^{2}\right\}
$$


This contribution comes entirely from Eq. (B.25) and the equality holds by virtue of the anomaly freedom condition (B.15). Dividing each of (B.22)-(B.28) by $(\sqrt{p} / \nu)\left(1+f_{3}\right) \dot{\varphi}$ and neglecting subleading orders of Planck's constant, we see that (B.21) reads

$$
\begin{aligned}
\delta \ddot{\varphi} & +2 \mathcal{H} \delta \dot{\varphi}\left(1+2 f_{3}^{\prime} p\right)+\nu \sigma\left(1-f_{3}\right) \nabla^{2} \delta \varphi+\nu p V_{, \varphi \varphi}(\varphi) \delta \varphi \\
& +2 \Phi\left[\nu p V_{, \varphi}(\varphi)\left(1+f_{1}\right)-\mathcal{H} \dot{\varphi}\left(f_{3}^{\prime} p\right)\right]-\dot{\varphi}\left[\left(1+f_{1}\right) \dot{\Phi}+3\left(1+g_{1}\right) \dot{\Psi}\right]=0,
\end{aligned}
$$

which is equivalent to the perturbed Klein-Gordon equation (B.9), since $\nu \sigma=\alpha^{2}$, and the coefficient in front of the $\delta \dot{\varphi}$-term in (B.29) can be rewritten, by virtue of (B.13)-(B.18), in several equivalent forms:

$$
1+2 f_{3}^{\prime} p=1-\frac{\nu^{\prime} p}{\nu}-g_{1}^{\prime} p=1+3\left(f-f_{3}\right)=1+\frac{\nu^{\prime} p}{\nu}+3 g_{1} .
$$

Note that the second expression coincides with the coefficient in (B.9).

It is also possible to derive an equation not containing matter fields by subtracting (B.6) divided by $\left(1+f_{3}\right)$ from (B.7). Eliminating $V_{, \varphi}, \dot{\varphi}^{2}$ and $\delta \varphi$ using the background Klein-Gordon equation (B.3), background Raychaudhuri equation (B.2) and the perturbed diffeomorphism equation (B.10) respectively one obtains

$$
\begin{aligned}
\ddot{\Psi} & -\alpha^{2}\left(1-f_{3}\right) \nabla^{2} \Psi+2 \dot{\Psi}\left[\mathcal{H}\left(1+f_{3}^{\prime} p+2 \frac{\nu^{\prime} p}{\nu}\right)-\frac{\ddot{\varphi}}{\dot{\varphi}}\right] \\
& +2 \Psi\left[\dot{\mathcal{H}}\left(1+\frac{2 \alpha^{\prime}}{\alpha}\right)+\mathcal{H}^{2}\left(2 \frac{\alpha^{\prime \prime} p^{2}}{\alpha}+2 \frac{\nu^{\prime} p}{\nu}+\left(f_{3}-f\right)^{\prime} p\right)-\mathcal{H} \frac{\ddot{\varphi}}{\dot{\varphi}}\left(1+\frac{2 \alpha^{\prime} p}{\alpha}\right)\right]=0 .
\end{aligned}
$$

In order to arrive at the last expression, the anomaly cancellation conditions (B.13), (B.14), (B.16), and (B.17) have been used, along with the fact that

$$
\left(\frac{\alpha^{\prime} p}{\alpha}\right)^{\prime} p=\frac{\alpha^{\prime \prime} p^{2}}{\alpha}+\frac{\alpha^{\prime} p}{\alpha}
$$

up to higher order terms in Planck's constant. Interestingly, Eq. (B.30) can also be obtained by replacing all the $\delta \varphi$ - and $\delta \dot{\varphi}$-terms in the perturbed Hamiltonian constraint equation (B.6) by the corresponding expressions according to (B.10). Consistency of the original equations demonstrated above ensures that one will arrive at the same equation (B.30).

As these explicit calculations demonstrate, consistency crucially relies on tight relationships between different corrections in the mode equations. Moreover, corrections in mode equations must be closely related to corrections in the background equations. These relationships follow from the condition of anomaly-freedom as realized by a closed constraint algebra. If one were to fix the gauge before quantization or to modify the equations for classical gauge-invariant variables, these tight relations would be overlooked and corrections would be much more ambiguous.

\section{References}

[1] P. A. M. Dirac, The theory of gravitation in Hamiltonian form, Proc. Roy. Soc. A 246 (1958) 333-343

[2] S. A. Hojman, K. Kuchař, and C. Teitelboim, Geometrodynamics Regained, Ann. Phys. (New York) 96 (1976) 88-135 
[3] K. V. Kuchař, Geometrodynamics regained: A Lagrangian approach, J. Math. Phys. 15 (1974) 708-715

[4] B. Dittrich and J. Tambornino, A perturbative approach to Dirac observables and their space-time algebra, Class. Quantum Grav. 24 (2007) 757-784, [gr-qc/0610060]

[5] B. Dittrich and J. Tambornino, Gauge invariant perturbations around symmetry reduced sectors of general relativity: applications to cosmology, Class. Quantum Grav. 24 (2007) 4543-4585, [gr-qc/0702093]

[6] K. Giesel, S. Hofmann, T. Thiemann, and O. Winkler, Manifestly Gauge-Invariant General Relativistic Perturbation Theory: I. Foundations, Class. Quantum Grav. 27 (2010) 055005, [arXiv:0711.0115]

[7] K. Giesel, S. Hofmann, T. Thiemann, and O. Winkler, Manifestly Gauge-Invariant General Relativistic Perturbation Theory: II. FRW Background and First Order, Class. Quantum Grav. 27 (2010) 055006, [arXiv:0711.0117]

[8] P. Malkiewicz, Reduced phase space approach to Kasner universe and the problem of time in quantum theory, Class. Quantum Grav. 29 (2012) 075008, [arXiv:1105.6030]

[9] A. Komar, Constraints, Hermiticity, and Correspondence, Phys. Rev. D 19 (1979) 2908-2912

[10] A. Komar, Consistent Factor Ordering Of General Relativistic Constraints, Phys. Rev. D 20 (1979) 830-833

[11] M. Bojowald, G. Hossain, M. Kagan, and S. Shankaranarayanan, Anomaly freedom in perturbative loop quantum gravity, Phys. Rev. D 78 (2008) 063547, [arXiv:0806.3929]

[12] J. D. Reyes, Spherically Symmetric Loop Quantum Gravity: Connections to 2-Dimensional Models and Applications to Gravitational Collapse, PhD thesis, The Pennsylvania State University, 2009

[13] T. Cailleteau, J. Mielczarek, A. Barrau, and J. Grain, Anomaly-free scalar perturbations with holonomy corrections in loop quantum cosmology, Class. Quantum Grav. 29 (2012) 095010, [arXiv:1111.3535]

[14] M. Bojowald and G. M. Paily, Deformed General Relativity and Effective Actions from Loop Quantum Gravity, Phys. Rev. D 86 (2012) 104018, [arXiv:1112.1899]

[15] R. Arnowitt, S. Deser, and C. W. Misner, The Dynamics of General Relativity, In L. Witten, editor, Gravitation: An Introduction to Current Research, Wiley, New York, 1962; Gen. Rel. Grav. 40 (2008) 1997-2027

[16] J. Schwinger, Quantized gravitational field, Phys. Rev. 130 (1962) 1253-1258

[17] A. Ashtekar, New Hamiltonian Formulation of General Relativity, Phys. Rev. D 36 (1987) 1587-1602

[18] J. F. Barbero G., Real Ashtekar Variables for Lorentzian Signature Space-Times, Phys. Rev. D 51 (1995) 5507-5510, [gr-qc/9410014]

[19] G. Immirzi, Real and Complex Connections for Canonical Gravity, Class. Quantum Grav. 14 (1997) L177-L181

[20] C. Rovelli, Quantum Gravity, Cambridge University Press, Cambridge, UK, 2004

[21] T. Thiemann, Introduction to Modern Canonical Quantum General Relativity, Cambridge University Press, Cambridge, UK, 2007, [gr-qc/0110034]

[22] A. Ashtekar and J. Lewandowski, Background independent quantum gravity: A status report, Class. Quantum Grav. 21 (2004) R53-R152, [gr-qc/0404018]

[23] M. Bojowald, Canonical Gravity and Applications: Cosmology, Black Holes, and Quantum Gravity, Cambridge University Press, Cambridge, 2010 
[24] M. Bojowald, G. Hossain, M. Kagan, and C. Tomlin, Quantum matter in quantum space-time, Quantum Matter 2 (2013) 436-443, [arXiv:1302.5695]

[25] C. Rovelli and L. Smolin, Loop Space Representation of Quantum General Relativity, Nucl. Phys. B 331 (1990) 80-152

[26] C. Rovelli and L. Smolin, Discreteness of Area and Volume in Quantum Gravity, Nucl. Phys. B 442 (1995) 593-619, [gr-qc/9411005], Erratum: Nucl. Phys. B 456 (1995) 753

[27] A. Ashtekar and J. Lewandowski, Quantum Theory of Geometry I: Area Operators, Class. Quantum Grav. 14 (1997) A55-A82, [gr-qc/9602046]

[28] C. Rovelli and L. Smolin, The physical Hamiltonian in nonperturbative quantum gravity, Phys. Rev. Lett. 72 (1994) 446-449, [gr-qc/9308002]

[29] T. Thiemann, Quantum Spin Dynamics (QSD), Class. Quantum Grav. 15 (1998) 839-873, [gr-qc/9606089]

[30] M. Bojowald, G. M. Paily, and J. D. Reyes, Discreteness corrections and higher spatial derivatives in effective canonical quantum gravity, [arXiv:1402.5130]

[31] M. Bojowald, Inverse Scale Factor in Isotropic Quantum Geometry, Phys. Rev. D 64 (2001) 084018, [gr-qc/0105067]

[32] M. Bojowald and G. Calcagni, Inflationary observables in loop quantum cosmology, JCAP 1103 (2011) 032, [arXiv:1011.2779]

[33] T. Thiemann, Anomaly-Free Formulation of Non-Perturbative, Four-Dimensional Lorentzian Quantum Gravity, Phys. Lett. B 380 (1996) 257-264, [gr-qc/9606088]

[34] J. Lewandowski and D. Marolf, Loop Constraints: A Habitat and their Algebra, Int. J. Mod. Phys. D 7 (1998) 299-330, [gr-qc/9710016]

[35] R. Gambini, J. Lewandowski, D. Marolf, and J. Pullin, On the Consistency of the Constraint Algebra in Spin Network Quantum Gravity, Int. J. Mod. Phys. D 7 (1998) 97-109, [gr-qc/9710018]

[36] H. Nicolai, K. Peeters, and M. Zamaklar, Loop quantum gravity: an outside view, Class. Quantum Grav. 22 (2005) R193-R247, [hep-th/0501114]

[37] A. Perez and D. Pranzetti, On the regularization of the constraints algebra of Quantum Gravity in $2+1$ dimensions with non-vanishing cosmological constant, Class. Quantum Grav. 27 (2010) 145009, [arXiv:1001.3292]

[38] A. Henderson, A. Laddha, and C. Tomlin, Constraint algebra in LQG reloaded : Toy model of a U(1) ${ }^{3}$ Gauge Theory I, Phys. Rev. D 88 (2013) 044028, [arXiv:1204.0211]

[39] A. Henderson, A. Laddha, and C. Tomlin, Constraint algebra in LQG reloaded : Toy model of an Abelian gauge theory - II Spatial Diffeomorphisms, Phys. Rev. D 88 (2013) 044029, [arXiv:1210.3960]

[40] C. Tomlin and M. Varadarajan, Towards an Anomaly-Free Quantum Dynamics for a Weak Coupling Limit of Euclidean Gravity, Phys. Rev. D 87 (2013) 044039, [arXiv:1210.6869]

[41] S. Brahma, Spherically symmetric canonical quantum gravity, [arXiv:1411.3661]

[42] G. J. Olmo and P. Singh, Covariant Effective Action for Loop Quantum Cosmology a la Palatini, JCAP 0901 (2009) 030, [arXiv:0806.2783]

[43] T. P. Sotiriou, Covariant Effective Action for Loop Quantum Cosmology from Order Reduction, Phys. Rev. D 79 (2009) 044035, [arXiv:0811.1799]

[44] K. Vandersloot, On the Hamiltonian Constraint of Loop Quantum Cosmology, Phys. Rev. D 71 (2005) 103506, [gr-qc/0502082] 
[45] A. Ashtekar, T. Pawlowski, and P. Singh, Quantum Nature of the Big Bang: Improved dynamics, Phys. Rev. D 74 (2006) 084003, [gr-qc/0607039]

[46] M. Bojowald, Large scale effective theory for cosmological bounces, Phys. Rev. D 75 (2007) 081301(R), [gr-qc/0608100]

[47] L. Linsefors and A. Barrau, Restrictions on curved cosmologies in modified gravity from metric considerations, [arXiv:1411.7258]

[48] C. P. Burgess, Quantum Gravity in Everyday Life: General Relativity as an Effective Field Theory, Living Rev. Relativity 7 (2004), [gr-qc/0311082], http://www.livingreviews.org/lrr-2004-5

[49] J. F. Donoghue, General relativity as an effective field theory: The leading quantum corrections, Phys. Rev. D 50 (1994) 3874-3888, [gr-qc/9405057]

[50] A. Ashtekar, J. Lewandowski, D. Marolf, J. Mourão, and T. Thiemann, Quantization of Diffeomorphism Invariant Theories of Connections with Local Degrees of Freedom, J. Math. Phys. 36 (1995) 6456-6493, [gr-qc/9504018]

[51] A. Laddha and M. Varadarajan, The Diffeomorphism Constraint Operator in Loop Quantum Gravity, [arXiv:1105.0636]

[52] P. Hořava, Quantum gravity at a Lifshitz point, Phys. Rev. D 79 (2009) 084008, [arXiv:0901.3775]

[53] M. Bojowald and A. Skirzewski, Effective Equations of Motion for Quantum Systems, Rev. Math. Phys. 18 (2006) 713-745, [math-ph/0511043]

[54] M. Bojowald and A. Skirzewski, Quantum Gravity and Higher Curvature Actions, Int. J. Geom. Meth. Mod. Phys. 4 (2007) 25-52, [hep-th/0606232], eConf C 0602061 (2006) 03

[55] M. Bojowald, S. Brahma, and E. Nelson, Higher time derivatives in effective equations of canonical quantum systems, Phys. Rev. D 86 (2012) 105004, [arXiv:1208.1242]

[56] M. Bojowald and A. Tsobanjan, Effective Casimir conditions and group coherent states, Class. Quantum Grav. 31 (2014) 115006, [arXiv:1401.5352]

[57] M. Bojowald and D. Simpson, Factor ordering and large-volume dynamics in quantum cosmology, ClassQuantum Grav. 31 (2014) 185016, [arXiv:1403.6746]

[58] M. Bojowald, B. Sandhöfer, A. Skirzewski, and A. Tsobanjan, Effective constraints for quantum systems, Rev. Math. Phys. 21 (2009) 111-154, [arXiv:0804.3365]

[59] M. Bojowald and A. Tsobanjan, Effective constraints for relativistic quantum systems, Phys. Rev. D 80 (2009) 125008, [arXiv:0906.1772]

[60] A. Ashtekar, M. Bojowald, and J. Lewandowski, Mathematical structure of loop quantum cosmology, Adv. Theor. Math. Phys. 7 (2003) 233-268, [gr-qc/0304074]

[61] V. Taveras, Corrections to the Friedmann Equations from LQG for a Universe with a Free Scalar Field, Phys. Rev. D 78 (2008) 064072, [arXiv:0807.3325]

[62] Y. Ding, Y. Ma, and J. Yang, Effective Scenario of Loop Quantum Cosmology, Phys. Rev. Lett. 102 (2009) 051301, [arXiv:0808.0990]

[63] M. Bojowald, The Semiclassical Limit of Loop Quantum Cosmology, Class. Quantum Grav. 18 (2001) L109-L116, [gr-qc/0105113]

[64] M. Bojowald, D. Brizuela, H. H. Hernandez, M. J. Koop, and H. A. Morales-Técotl, High-order quantum back-reaction and quantum cosmology with a positive cosmological constant, Phys. Rev. D 84 (2011) 043514, [arXiv:1011.3022]

[65] M. Bojowald, Quantization ambiguities in isotropic quantum geometry, Class. Quantum Grav. 19 (2002) 5113-5130, [gr-qc/0206053] 
[66] M. Bojowald, H. Hernández, M. Kagan, and A. Skirzewski, Effective constraints of loop quantum gravity, Phys. Rev. D 75 (2007) 064022, [gr-qc/0611112]

[67] M. Bojowald, G. Calcagni, and S. Tsujikawa, Observational test of inflation in loop quantum cosmology, JCAP 11 (2011) 046, [arXiv:1107.1540]

[68] J. Brunnemann and T. Thiemann, Unboundedness of Triad-Like Operators in Loop Quantum Gravity, Class. Quantum Grav. 23 (2006) 1429-1483, [gr-qc/0505033]

[69] M. Bojowald and H. A. Kastrup, Symmetry Reduction for Quantized Diffeomorphism Invariant Theories of Connections, Class. Quantum Grav. 17 (2000) 3009-3043, [hep-th/9907042]

[70] M. Bojowald, Isotropic Loop Quantum Cosmology, Class. Quantum Grav. 19 (2002) 2717-2741, [gr-qc/0202077]

[71] M. Bojowald, Homogeneous loop quantum cosmology, Class. Quantum Grav. 20 (2003) 2595-2615, [gr-qc/0303073]

[72] M. Bojowald, Consistent Loop Quantum Cosmology, Class. Quantum Grav. 26 (2009) 075020, [arXiv:0811.4129]

[73] M. Bojowald, Loop quantum cosmology and inhomogeneities, Gen. Rel. Grav. 38 (2006) 1771-1795, [gr-qc/0609034]

[74] M. Bojowald, The dark side of a patchwork universe, Gen. Rel. Grav. 40 (2008) 639-660, [arXiv:0705.4398]

[75] M. Bojowald, Mathematical structure of loop quantum cosmology: Homogeneous models, SIGMA 9 (2013) 082, [arXiv:1206.6088]

[76] M. Bojowald, G. Calcagni, and S. Tsujikawa, Observational constraints on loop quantum cosmology, Phys. Rev. Lett. 107 (2011) 211302, [arXiv:1101.5391]

[77] M. Bojowald and S. Brahma, Effective constraint algebras with structure functions, [arXiv:1407.4444]

[78] N. Deruelle, M. Sasaki, Y. Sendouda, and D. Yamauchi, Hamiltonian formulation of f(Riemann) theories of gravity, Prog. Theor. Phys. 123 (2009) 169-185, [arXiv:0908.0679]

[79] T. Cailleteau and A. Barrau, Gauge invariance in Loop Quantum Cosmology: Hamilton-Jacobi and Mukhanov-Sasaki equations for scalar perturbations, [arXiv:1111.7192]

[80] T. Cailleteau, A. Barrau, J. Grain, and F. Vidotto, Consistency of holonomy-corrected scalar, vector and tensor perturbations in Loop Quantum Cosmology, Phys. Rev. D 86 (2012) 087301, [arXiv:1206.6736]

[81] T. Cailleteau, L. Linsefors, and A. Barrau, Anomaly-free perturbations with inverse-volume and holonomy corrections in Loop Quantum Cosmology, [arXiv:1307.5238]

[82] M. Bojowald, Spherically Symmetric Quantum Geometry: States and Basic Operators, Class. Quantum Grav. 21 (2004) 3733-3753, [gr-qc/0407017]

[83] K. Banerjee, G. Calcagni, and Martín-Benito, Introduction to loop quantum cosmology, SIGMA 8 (2012) 016, [arXiv:1109.6801]

[84] M. Bojowald, G. Hossain, M. Kagan, and S. Shankaranarayanan, Gauge invariant cosmological perturbation equations with corrections from loop quantum gravity, Phys. Rev. D 79 (2009) 043505, [arXiv:0811.1572]

[85] I. Agulló, A. Ashtekar, and W. Nelson, An Extension of the Quantum Theory of Cosmological Perturbations to the Planck Era, Phys. Rev. D 87 (2013) 043507, [arXiv:1211.1354]

[86] L.-F. Li, R.-G. Cai, Z.-K. Guo, and B. Hu, Non-Gaussian features from the inverse volume corrections in loop quantum cosmology, Phys. Rev. D 86 (2012) 044020, [arXiv:1112.2785] 
[87] J. M. Pons, D. C. Salisbury, and L. C. Shepley, Gauge transformations in the Lagrangian and Hamiltonian formalisms of generally covariant theories, Phys. Rev. D 55 (1997) 658-668, [gr-qc/9612037]

[88] M. Bojowald, J. D. Reyes, and R. Tibrewala, Non-marginal LTB-like models with inverse triad corrections from loop quantum gravity, Phys. Rev. D 80 (2009) 084002, [arXiv:0906.4767]

[89] R. Tibrewala, Inhomogeneities, loop quantum gravity corrections, constraint algebra and general covariance, Class. Quantum Grav. 31 (2014) 055010, [arXiv:1311.1297]

[90] V. Bonzom and B. Dittrich, Dirac's discrete hypersurface deformation algebras, Class. Quantum Grav. 30 (2013) 205013, [arXiv:1304.5983]

[91] E. Wilson-Ewing, Holonomy Corrections in the Effective Equations for Scalar Mode Perturbations in Loop Quantum Cosmology, Class. Quantum Grav. 29 (2012) 085005, [arXiv:1108.6265]

[92] A. Connes, Formule de trace en geometrie non commutative et hypothese de Riemann, C.R. Acad. Sci. Paris 323 (1996) 1231-1235

[93] S. Doplicher, K. Fredenhagen, and J. E. Roberts, The quantum structure of spacetime at the Planck scale and quantum fields, Commun. Math. Phys. 172 (1995) 187-220, [hep-th/0303037]

[94] G. Calcagni, Fractal universe and quantum gravity, Phys. Rev. Lett. 104 (2010) 251301, [arXiv:0912.3142]

[95] G. Calcagni, Multi-scale gravity and cosmology, JCAP 12 (2013) 041, [arXiv:1307.6382]

[96] M. Bojowald and G. M. Paily, Deformed General Relativity, Phys. Rev. D 87 (2013) 044044, [arXiv:1212.4773]

[97] G. Amelino-Camelia, Relativity: Special treatment, Nature 418 (2002) 34-35

[98] J. Magueijo and L. Smolin, Lorentz Invariance with an Invariant Energy Scale, Phys. Rev. Lett. 88 (2002) 190403

[99] J. Kowalski-Glikman, Introduction to Doubly Special Relativity, Lect. Notes Phys. 669 (2005) 131-159, [hep-th/0405273]

[100] D. Kovacevic, S. Meljanac, A. Pachol, and R. Strajn, Generalized Poincare algebras, Hopf algebras and kappa-Minkowski spacetime, Phys. Lett. B 711 (2012) 122-127, [arXiv:1202.3305]

[101] G. F. R. Ellis, A. Sumeruk, D. Coule, and C. Hellaby, Change of signature in classical relativity, Class. Quantum Grav. 9 (1992) 1535-1554

[102] L. Castelló Gomar and G. A. Mena Marugán, Uniqueness of the Fock quantization of scalar fields and processes with signature change in cosmology, [arXiv:1403.6984]

[103] G. Calcagni, B. de Carlos, and A. de Felice, Ghost conditions for Gauss-Bonnet cosmologies, Nucl. Phys. B 752 (2006) 404, [hep-th/0604201]

[104] M. Bojowald, Fluctuation energies in quantum cosmology, Phys. Rev. D 89 (2014) 124031, [arXiv:1404.5284]

[105] F. Girelli, F. Hinterleitner, and S. A. Major, Loop Quantum Gravity Phenomenology: Linking Loops to Observational Physics, SIGMA 8 (2012) 098, [arXiv:1210.1485]

[106] G. Calcagni, Observational Effects from Quantum Cosmology, Annalen Phys. 525 (2012) 323-338; A165, [arXiv:1209.0473]

[107] A. Barrau, T. Cailleteau, J. Grain, and J. Mielczarek, Observational issues in loop quantum cosmology, Class. Quantum Grav. 31 (2014) 053001, [arXiv:1309.6896]

[108] J. Mielczarek, Gravitational waves from the Big Bounce, JCAP 0811 (2008) 011, [arXiv:0807.0712] 
[109] A. Barrau and J. Grain, Cosmological footprint of loop quantum gravity, Phys. Rev. Lett. 102 (2009) 081301, [arXiv:0902.0145]

[110] J. Mielczarek, Tensor power spectrum with holonomy corrections in LQC, Phys. Rev. D 79 (2009) 123520, [arXiv:0902.2490]

[111] J. Mielczarek, T. Cailleteau, J. Grain, and A. Barrau, Inflation in loop quantum cosmology: Dynamics and spectrum of gravitational waves, Phys. Rev. D 81 (2010) 104049, [arXiv:1003.4660]

[112] J. Grain, A. Barrau, T. Cailleteau, and J. Mielczarek, Observing the Big Bounce with Tensor Modes in the Cosmic Microwave Background: Phenomenology and Fundamental LQC Parameters, Phys. Rev. D 82 (2010) 123520, [arXiv:1011.1811]

[113] L. Linsefors, T. Cailleteau, A. Barrau, and J. Grain, Primordial tensor power spectrum in holonomy corrected Omega-LQC, Phys. Rev. D 87 (2013) 107503, [arXiv:1212.2852]

[114] M. Martín-Benito, L. J. Garay, and G. A. Mena Marugán, Hybrid Quantum Gowdy Cosmology: Combining Loop and Fock Quantizations, Phys. Rev. D 78 (2008) 083516, [arXiv:0804.1098]

[115] R. H. Gowdy, Vacuum spacetimes with two-parameter spacelike isometry groups and compact invariant hypersurfaces: Topologies and boundary conditions, Ann. Phys. 83 (1974) 203-241

[116] J. Isenberg and V. Moncrief, Asymptotic behavior of the gravitational field and the nature of singularities in Gowdy space-times, Annals Phys. 199 (1990) 84-122

[117] B. Berger, Quantum Cosmology: Exact Solution For The Gowdy $T^{3}$ Model, Phys. Rev. D 11 (1975) 2770-2780

[118] A. Corichi, J. Cortez, G. A. Mena Marugan, and J. M. Velhinho, Quantum Gowdy $T^{3}$ Model: Schrodinger Representation with Unitary Dynamics, Phys. Rev. D 76 (2007) 124031, [arXiv:0710.0277]

[119] J. Cortez and G. A. Mena Marugan, Feasibility of a Unitary Quantum Dynamics in the Gowdy $T^{3}$ Cosmological Model, Phys. Rev. D 72 (2005) 064020, [gr-qc/0507139]

[120] J. Cortez, G. A. Mena Marugan, J. Olmedo, and J. M. Velhinho, Uniqueness of the Fock quantization of fields with unitary dynamics in nonstationary spacetimes, Phys. Rev. D 83 (2011) 025002, [arXiv:1101.2397]

[121] K. Banerjee and G. Date, Loop quantization of polarized Gowdy model on $T^{3}$ : classical theory, Class. Quantum Grav. 25 (2008) 105014, [arXiv:0712.0683]

[122] K. Banerjee and G. Date, Loop Quantization of polarized Gowdy model on $T^{3}$ : kinematical states and constraint operators, Class. Quantum Grav. 25 (2008) 145004, [arXiv:0712.0687]

[123] R. Haag, Local Quantum Physics, Springer-Verlag, Berlin, Heidelberg, New York, 1992

[124] M. Fernández-Méndez, G. A. Mena Marugán, and J. Olmedo, Hybrid quantization of an inflationary model: The flat case, Phys. Rev. D 88 (2013) 044013, [arXiv:1307.5222]

[125] D. Brizuela, G. A. Mena Marugán, and T. Pawlowski, Big Bounce and inhomogeneities, Class. Quantum Grav. 27 (2010) 052001, [arXiv:0902.0697]

[126] A. Ashtekar, W. Kaminski, and J. Lewandowski, Quantum field theory on a cosmological, quantum space-time, Phys. Rev. D 79 (2009) 064030, [arXiv:0901.0933]

[127] A. Dapor, J. Lewandowski, and J. Puchta, QFT on quantum spacetime: a compatible classical framework, [arXiv:1302.3038]

[128] M. Bojowald, The Universe: A View from Classical and Quantum Gravity, Wiley, Berlin, 2012 
[129] J. Mielczarek, Inflationary power spectra with quantum holonomy corrections, [arXiv:1311.1344]

[130] P. Diener, B. Gupt, M. Megevand, and P. Singh, Numerical evolution of squeezed and non-Gaussian states in loop quantum cosmology, Class. Quant. Grav. 31 (2014) 165006 [arXiv:1406.1486]. 